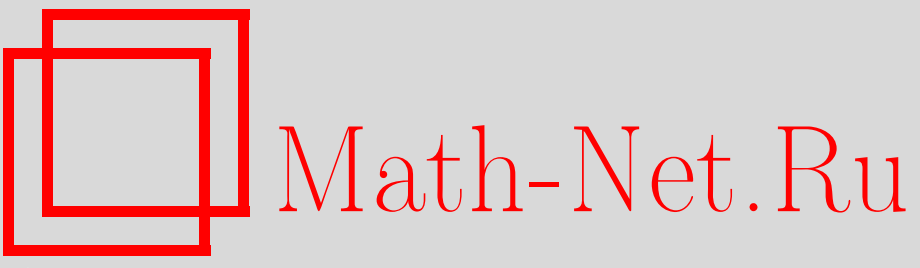

A. G. Chentsov, A. P. Baklanov, On an asymptotic analysis problem related to the construction of an attainability domain, Trudy Mat. Inst. Steklova, 2015, Volume 291, 292-311

DOI: https://doi.org/10.1134/S0371968515040226

Use of the all-Russian mathematical portal Math-Net.Ru implies that you have read and agreed to these terms of use

http://www.mathnet.ru/eng/agreement

Download details:

IP: 54.172 .240 .79

April 26, 2023, 10:35:51 


\title{
Об одной задаче асимптотического анализа, связанной с построением области достижимости ${ }^{1}$
}

\author{
А. Г. Ченцов ${ }^{2,3}$, А. П. Бакланов ${ }^{2,4}$ \\ Поступило 15 октября 2014 г.
}

\begin{abstract}
Задачи о построении и исследовании свойств областей достижимости играют важную роль в теории управления и ее приложениях. В частности, это относится к управлению при ограничениях импульсного характера, отражающих энергетику процесса. Ситуация осложняется возможной неустойчивостью процесса при изменении (в частности, при ослаблении) ограничений, связанных с краевыми и промежуточными условиями. Устойчивость задачи отсутствует, вообе говоря, и при ослаблении фазовых ограничений. В этих случаях естественно ориентироваться на асимптотический вариант постановки; это тем более целесообразно в условиях, когда приходится иметь дело с изначально асимптотическими требованиями. Во всех этих случаях представляется целесообразным использовать аналоги приближенных решений Дж. Варги. В то же время для поиска нужных приближенных (а по сути, асимптотических) решений естественно использовать обобщенные режимы, которые для задач с импульсными ограничениями и разрывностью в коэффициентах при управляющих воздействиях уже в классе линейных систем приводят к эффектам, имеющим смысл произведения разрывной функции на обобщенную. В большой серии работ одного из авторов для преодоления возникающих при этом трудностей были использованы конструкции расширения в классе конечно аддитивных мер. Настоящая работа следует данному подходу и в смысле своей идейной направленности отвечает инженерной задаче управления силой тяги двигателя в условиях заданной программы изменения его ориентации; при этом постулируется полное расходование энергоресурса в естественном (для целого ряда задач импульсного управления) режиме "узких" импульсов: множество моментов времени, для которых мгновенное управление отлично от нуля, допускает вложение в промежуток исчезающе малой длины. За это "короткое" время надо израсходовать весь энергоресурс, соблюдая с высокой степенью точности некоторые другие ограничения (по смыслу - моментные). При осуществлении этого следует учитывать возможную разрывность зависимостей, определяющих коэффициенты при управляющих воздействиях. В качестве естественного аналога области достижимости в работе используется множество притяжения, построение которого вместе с последующим изучением основных его свойств составляет цель настоящей работы.
\end{abstract}

DOI: $10.1134 /$ S0371968515040226

\section{1. ВВЕДЕНИЕ}

Рассматривается задача, связанная по смыслу с построением и исследованием свойств области достижимости (ОД) линейной управляемой системы с возможной разрывностью в коэффициентах при управляющих воздействиях. Предполагается, что выбор программного управления стеснен ограничениями импульсного характера и (дополнительно) условиями, имеющими

\footnotetext{
${ }^{1}$ Работа выполнена при финансовой поддержке Российского фонда фундаментальных исследований (проекты 12-01-00537a, 13-01-90414-Укр_ф_а, 13-01-00304а) и программ фундаментальных исследований Президиума РАН (проекты 12-П-1-1012, 12-П-1-1019).

${ }^{2}$ Институт математики и механики им. Н.Н. Красовского УрО РАН, Екатеринбург, Россия; Уральский федеральный университет им. первого Президента России Б.Н. Ельцина, Екатеринбург, Россия.

${ }^{3}$ E-mail: chentsov@imm.uran.ru

${ }^{4}$ International Institute for Applied Systems Analysis, Laxenburg, Austria.

E-mail: artem.baklanov@gmail.com
} 
смысл обеспечения исчезающе малой продолжительности импульса, а также ограничениями моментного характера. Итак, обсуждаются проблемы достижимости в условиях весьма различных ограничений, некоторые из которых изначально имеют асимптотический характер.

Ясно, что вопросы построения ОД имеют важное значение для теории оптимального управления. Так, например, в задаче с фиксированным моментом окончания оптимизация (терминального) критерия связана с перебором точек ОД, на которых вычисляются значения функции стоимости; при изменении ОД за счет возмущения системы ограничений, естественно, могут изменяться экстремум и реализующие его управления. Создание и развитие теории управления неразрывно связано с именем Л.С. Понтрягина, что нашло свое отражение в выдающемся достижении - принципе максимума Понтрягина (см. [35]).

Вместе с тем в задаче о построении ОД зачастую отсутствует устойчивость при возмущении, и в частности при ослаблении, ограничений. Выделяя для дальнейшего рассмотрения последний случай, отметим, однако, что упомянутую неустойчивость можно использовать "в положительном направлении", ориентируясь на реализацию решений "на грани фола". Само же нахождение таких решений и возникающих при их использовании возможностей традиционно связывается с идеей расширения исходной задачи, при котором пространство обычных управлений погружается в компакт в виде всюду плотного множества (см. [27, 36]). Элементы упомянутого компакта можно рассматривать в качестве обобщенных управлений (ОУ); в $[27,36]$ эти элементы отождествляются с мерозначными функциями (времени). Аналогичные конструкции в игровых задачах динамики широко использовались Н.Н. Красовским и его учениками (см. [30, 31, 34]).

В связи с задачами импульсного управления отметим оригинальный подход Н.Н. Красовского, связанный с применением аппарата теории обобщенных функций (см. [29]) и послуживший основой целого ряда направлений в исследовании этого круга задач.

В настоящей работе развивается подход, связанный с расширением абстрактных задач управления в классе конечно аддитивных (к.а.) мер (см. $[5-7,9,23]$ и др.); используются также более поздние конструкции, связанные с применением в качестве обобщенных элементов ультрафильтров (у/ф) [2, гл. I] широко понимаемых измеримых пространств (см. $[11-15,17,18$, 21]). Учитываются положения [13], касающиеся представления компакта Стоуна (пространство у/ф алгебры множеств) для одной естественной версии измеримого пространства, "единица" которого соответствует промежутку управления. Наконец, используется двойственность [19] у/ф алгебры множеств и к.а. $(0,1)$-мер на данной алгебре. Статья является продолжением работы [22].

Рассмотрим на содержательном уровне вариант исследуемой ниже задачи. Предполагая выполненным, если потребуется, неособое линейное преобразование [31, с. 160] и имея в виду проблему построения ОД в заданный момент времени, рассмотрим линейную систему

$$
\dot{x}(t)=u(t) \mathbf{b}(t)+\mathbf{c}(t)
$$

в $m$-мерном фазовом пространстве на промежутке $I_{0} \triangleq\left[t_{0}, \vartheta_{0}\right]\left(t_{0}<\vartheta_{0}\right)$; здесь $u=u(\cdot)-$ скалярная управляющая функция, $\mathbf{a} \mathbf{b}=\mathbf{b}(\cdot)$ и $\mathbf{c}=\mathbf{c}(\cdot)$ суть $m$-вектор-функции на $I_{0}$. При этом $u \in U$, где $U$ - заданное множество; $x\left(t_{0}\right)=x_{0}$, где $x_{0}$ - заданный $m$-мерный вектор. Нас интересуют возможные состояния $x\left(\vartheta_{0}\right)$. Будем полагать, что $U-$ множество всех неотрицательных кусочно постоянных (к.п.) функций на $I_{0}$, у которых импульс силы (интеграл на $I_{0}$ ) равен заданному положительному числу $\mathbf{d}$, что означает по смыслу запас топлива, которое должно быть полностью израсходовано. Полагаем, что компоненты $m$-вектор-функций $\mathbf{b}$ и с являются равномерными пределами к.п. вещественнозначных $($ в $/ 3)$ функций на $I_{0} ; \mathbf{b}(t)$ определяет ориентацию двигателя в каждый момент времени $t \in I_{0}$ и, возможно, вырабатывается другой подсистемой, как и $\mathbf{c}(t)$. Не ограничивая общности, можно полагать $\mathbf{d}=1$, осуществляя, если это не так, перенормировку $\mathbf{b .}$ 
Пусть выбор $u \in U$ стеснен некоторыми дополнительными ограничениями. Прежде всего будем требовать, чтобы управление $u=u(\cdot)$ формировалось как импульс исчезающе малой продолжительности, что естественно для импульсного управления и мотивируется, в частности, задачами космической навигации [25] (соображения, связанные с энергетикой процесса). Кроме того, будем полагать, что управление $u \in U$ должно удовлетворять некоторым ограничениям моментного характера. Последние могут получаться переформулировкой краевых и промежуточных условий, но могут иметь и другой смысл (см. [5, Sect. 1.3]). Так или иначе, возникает условие на выбор $u \in U$, имеющее вид

$$
\int_{t_{0}}^{\vartheta_{0}} u(t) \mathbf{r}(t) d t \in \mathbf{X},
$$

где $\mathbf{r}$ есть $m$-вектор-функция, все компоненты которой являются равномерными пределами к.п. в/з функций на $I_{0}$, а $\mathbf{X}$ - замкнутое множество в $m$-мерном пространстве. Рассматриваем проблему приближенного соблюдения (1.2) в режиме "узких импульсов". При этом допускаем различные варианты ослабления $\mathbf{X}$-ограничения (1.2), а именно сравниваем "полноразмерное" ослабление (1.2) и ослабление данного ограничения по части координат. В последнем случае требуем, чтобы компоненты $\mathbf{r}$, отвечающие прочим координатам (вдоль которых ослабление не производится), были к.п. функциями. Здесь реализуется, таким образом, усложненный (за счет применения только "узких импульсов") вариант условий частичной ступенчатости интегранта при управлении, которые детально исследовались ранее в $[5-7,9,23]$ и целом ряде других работ. Для данной усложненной постановки будет установлено существование универсального (относительно упомянутых вариантов ослабления ограничений) множества притяжения (МП), реализуемого в режиме "узких импульсов". Фактически речь идет об установлении эквивалентности двух вариантов ограничений асимптотического характера (OAX). Данная эквивалентность заключается в совпадении МП, которые могут рассматриваться в качестве естественных асимптотических аналогов ОД (подчеркнем, что одна из компонент обоих вариантов ОАХ - режим "узких импульсов" - изначально является условием на асимптотику программных управлений). Применительно к системе (1.1) мы рассматриваем МП на значениях вектор-функционала

$$
u \mapsto \int_{t_{0}}^{\vartheta_{0}} u(t) \mathbf{b}(t) d t
$$

что вполне достаточно для целей описания асимптотики соответствующих множеств терминальных состояний (исходная постановка может быть усложнена; так, например, можно было бы рассматривать элементы притяжения в пространстве траекторий с топологией поточечной сходимости, при этом саму систему уже не имело бы смысла сводить к виду (1.1) на основе преобразования [31, с. 160]; см. в этой связи абстрактные варианты “асимптотической" постановки в $[6,7,9,23])$. Упомянутое универсальное МП будет построено в терминах отображений $\mathbf{b}$ и $\mathbf{r}$ (см. (1.1), (1.2)); см. в этой связи разд. 6. Ключевую роль играет здесь (также универсальное) вспомогательное МП в пространстве ОУ, определяемых в виде к.а. мер.

В связи с (1.3) отметим еще одну гипотетическую интерпретацию, допускающую аналогию с $[4,8]$ и уже не связанную непосредственно с системой (1.1). Итак, полагая вектор-функции $\mathbf{b}$ и $\mathbf{r}$ заданными, можно рассматривать их компоненты в виде некоторых случайных величин (CB), а операции интегрирования в (1.2), (1.3) интерпретировать как вычисление математических ожиданий (МО) относительно вероятностных распределений с вырождающейся плотностью (снова имеем режим “узких импульсов"). Теперь уже (1.3) можно трактовать как 
сопоставление распределению вектора МО заданного набора СВ, т.е. как проблему достижимости в $m$-мерном пространстве на значениях МО при условии, что выбор самого распределения удовлетворяет ограничениям, одно из которых - "узкие импульсы" - уже оговаривалось ранее, а второе, связанное с (1.2), сводится к условию на МO каких-то других $\mathrm{CB}$, составляющих в совокупности случайный вектор $\mathbf{r}$. Фиксируя естественную алгебру множеств промежутка $I_{0}$, упомянутые содержательные построения можно сделать строгими, действуя по аналогии с $[4,8]$, после чего снова ввести ОАХ уже на выбор плотности вероятности $(u=u(\cdot)$ в $(1.3))$. В такой редакции конструируемое далее расширение задачи также реализуется в классе к.а. вероятностей со свойством слабой абсолютной непрерывности относительно соответствующего сужения меры Лебега (напомним об условии нормировки $u=u(\cdot)$ в (1.1)).

Таким образом, имеет смысл дать изложение конструкций в форме, пригодной для использования в различных содержательных задачах, для которых сохраняет смысл идея достижимости при ОАХ. Упомянутая "широкая" постановка задачи о достижимости в применении к задачам оптимизации (о чем говорилось ранее) допускает естественную связь с проблемой моментов $[29, \S 16]$. В то же время линейность зависимости (1.3), строго говоря, не обязательна в рассматриваемом асимптотическом варианте задачи о достижимости: допускаются, в частности, нелинейные безынерционные преобразования типа [6, (3.10.4), (3.10.5)] и более общие непрерывные преобразования вида $[6,(3.10 .3)]$. В настоящей работе такие вопросы не рассматриваются; излагаемые ниже (более частные) конструкции ориентированы на получение конкретных способов реализации МП.

\section{2. ОБЩИЕ ОБОЗНАЧЕНИЯ И ОПРЕДЕЛЕНИЯ}

Используется обычная теоретико-множественная символика (кванторы, связки и т.п.); $\triangleq$ обозначает равенство по определению. Принимаем аксиому выбора; семейством называем множество, все элементы которого сами являются множествами. Далее, как правило, рассматриваются семейства подмножеств (п/м) того или иного фиксированного множества. Если $y$ и $z$ - объекты, то через $\{y ; z\}$ обозначим множество, содержащие $y, z$ и не содержащее никаких других элементов. Тогда $\{y\} \triangleq\{y ; y\}$ есть одноэлементное множество, содержащее объект $y$, а $(p, q) \triangleq\{\{p\} ;\{p ; q\}\}$ - упорядоченная пара (УП) с первым элементом $p$ и вторым элементом $q$. Для всякой УП $z$ через $\operatorname{pr}_{1}(z)$ и $\operatorname{pr}_{2}(z)$ условимся обозначать соответственно первый и второй элементы $z$, однозначно определяемые условием $z=\left(\operatorname{pr}_{1}(z), \operatorname{pr}_{2}(z)\right)$. Через $\mathcal{P}(X)\left(\mathcal{P}^{\prime}(X)\right)$ обозначаем семейство всех (всех непустых) п/м множества $X$. Через $B^{A}$ обозначаем $\left[32\right.$, гл. II, §6] множество всех отображений из множества $A$ в множество $B$; если $f \in B^{A}$ и $C \in \mathcal{P}(A)$, то $f^{1}(C) \triangleq\{f(x): x \in C\}$ есть образ $C$ при действии $f$. Как обычно, $\mathbb{R}-$ вещественная прямая, $\mathbb{N} \triangleq\{1 ; 2 ; \ldots\}$ и $\overline{1, m} \triangleq\{k \in \mathbb{N} \mid k \leq m\}$ для $m \in \mathbb{N}$. Полагая, что элементы $\mathbb{N}$ не являются множествами, следуем обычному соглашению: для всяких множества $T$ и числа $k \in \mathbb{N}$ вместо $T^{\overline{1, k}}$ пишем $T^{k}$.

Специальные семейства. В пределах данного пункта фиксируем непустое множество $E$, получая в виде $\mathcal{P}^{\prime}(\mathcal{P}(E))$ семейство всех непустых подсемейств $\mathcal{P}(E)$ и

$$
\pi[E] \triangleq\left\{\mathcal{E} \in \mathcal{P}^{\prime}(\mathcal{P}(E)) \mid(\varnothing \in \mathcal{E}) \&(E \in \mathcal{E}) \&(A \cap B \in \mathcal{E} \forall A, B \in \mathcal{E})\right\}
$$

(семейство всех $\pi$-систем [3, с. 14] п/м $E$ с “нулем" и "единицей”),

$$
(\operatorname{alg})[E] \triangleq\{\mathcal{L} \in \pi[E] \mid E \backslash L \in \mathcal{L} \forall L \in \mathcal{L}\}, \quad(\text { top })[E] \triangleq\left\{\tau \in \pi[E] \mid \bigcup_{G \in \mathcal{G}} G \in \tau \forall \mathcal{G} \in \mathcal{P}^{\prime}(\tau)\right\}
$$

(семейства всех алгебр п/м $E$ и всех топологий на $E$ соответственно). В виде

$$
\beta[E] \triangleq\left\{\mathcal{S} \in \mathcal{P}^{\prime}(\mathcal{P}(E)) \mid \forall B_{1}, B_{2} \in \mathcal{S} \exists B_{3} \in \mathcal{S}: B_{3} \subset B_{1} \cap B_{2}\right\}
$$


имеем семейство всех непустых направленных подсемейств $\mathcal{P}(E)$, а в виде $\beta_{0}[E] \triangleq\{\mathcal{B} \in \beta[E] \mid$ $\varnothing \notin \mathcal{B}\}-$ семейство всех баз фильтров (БФ) множества $E$. До конца настоящего пункта фиксируем $\pi$-систему $\mathcal{E} \in \pi[E]$. Тогда

$$
\mathbb{F}^{*}(\mathcal{E}) \triangleq\left\{\mathcal{F} \in \mathcal{P}^{\prime}(\mathcal{E} \backslash\{\varnothing\}) \mid(A \cap B \in \mathcal{F} \forall A, B \in \mathcal{F}) \&(\forall F \in \mathcal{F} \forall L \in \mathcal{E}(F \subset L) \Rightarrow(L \in \mathcal{F}))\right\}
$$

есть множество всех фильтров $\pi$-системы $\mathcal{E}$, а

$$
\mathbb{F}_{0}^{*}(\mathcal{E}) \triangleq\left\{\mathcal{U} \in \mathbb{F}^{*}(\mathcal{E}) \mid \forall \mathcal{F} \in \mathbb{F}^{*}(\mathcal{E})(\mathcal{U} \subset \mathcal{F}) \Rightarrow(\mathcal{U}=\mathcal{F})\right\}
$$

есть (непустое) множество всех у/ф $\mathcal{E}$. При этом

$$
\left(E \text {-fi) }[\mathcal{B} \mid \mathcal{E}] \triangleq\{L \in \mathcal{E} \mid \exists B \in \mathcal{B}: B \subset L\} \in \mathbb{F}^{*}(\mathcal{E}) \quad \forall \mathcal{B} \in \beta_{0}[E]\right.
$$

(согласно $(2.1)$ БФ $E$ порождают фильтры $\mathcal{E}$ ). Введем также $\beta_{\mathcal{E}}^{0}[E] \triangleq\left\{\mathcal{B} \in \beta_{0}[E] \mid \mathcal{B} \subset \mathcal{E}\right\}$; семейства из $\beta_{\mathcal{E}}^{0}[E]$ могут использоваться в $(2.1)$. Пусть $\beta_{\mathcal{E}}^{00}[E] \triangleq\left\{\mathcal{B} \in \beta_{\mathcal{E}}^{0}[E] \mid\left(E\right.\right.$-fi) $\left.[\mathcal{B} \mid \mathcal{E}] \in \mathbb{F}_{0}^{*}(\mathcal{E})\right\}$ (семейство БФ, содержащихся в $\mathcal{E}$ и порождающих у/ф этой $\pi$-системы). Заметим, что $((E, \mathcal{E})$-ult $)[x] \triangleq\{L \in \mathcal{E} \mid x \in L\} \in \mathbb{F}^{*}(\mathcal{E})$ при $x \in E$.

Элементы топологии. В пределах данного пункта фиксируем топологию $\tau \in($ top $)[E]$, получая в виде $(E, \tau)$ топологическое пространство (ТП). Если $x \in E$, то $N_{\tau}^{0}(x) \triangleq\{G \in \tau \mid$ $x \in G\} \in \mathbb{F}^{*}(\tau)$; в частности, $N_{\tau}^{0}(x) \in \beta_{0}[E]$ порождает фильтр окрестностей $N_{\tau}(x) \triangleq$ $\triangleq(E$-fi $)\left[N_{\tau}^{0}(x) \mid \mathcal{P}(E)\right] \in \mathbb{F}[E]$, где $\mathbb{F}[E] \triangleq \mathbb{F}^{*}(\mathcal{P}(E))$ соответствует определениям [2, гл. I]. Через $(\tau$-comp) $[E]$ обозначаем семейство всех непустых компактных в ТП $(E, \tau)$ п/м $E$ (см. [26, c. 196]). Если $J \in \mathcal{P}(E)$, то $\operatorname{cl}(J, \tau)$ есть по определению замыкание $J$ в ТП $(E, \tau)$. Если $S \in \mathcal{P}[E]$, то $\left.\tau\right|_{S} \triangleq\{S \cap G: G \in \tau\} \in(\operatorname{top})[S]$ есть топология, индуцированная в $S$ из $(E, \tau)$.

Непрерывность. Если $\left(X, \tau_{1}\right)$ и $\left(Y, \tau_{2}\right)$ суть ТП, $X \neq \varnothing, Y \neq \varnothing$, то $C\left(X, \tau_{1}, Y, \tau_{2}\right) \triangleq$ $\triangleq\left\{g \in Y^{X} \mid g^{-1}(G) \in \tau_{1} \forall G \in \tau_{2}\right\}$ (множество всех непрерывных отображений из $\left(X, \tau_{1}\right)$ в $\left.\left(Y, \tau_{2}\right)\right)$.

\section{3. МНОЖЕСТВА ПРИТЯЖЕНИЯ (КРАТКИЕ СВЕДЕНИЯ)}

Ниже используется "обычная" сходимость по Мору-Смиту [28, гл. 2]. Направленностью в множестве $S$ называем триплет $(D, \preceq, g)$, где $\preceq-$ направление на непустом множестве $D$ и $g \in S^{D}$;

$$
(S \text {-ass })[D ; \preceq ; g] \triangleq\{H \in \mathcal{P}(S) \mid \exists d \in D \forall \delta \in D(d \preceq \delta) \Rightarrow(g(\delta) \in H)\} \in \mathbb{F}[S]
$$

(фильтр, ассоциированный с направленностью) и при $\tau \in(\mathrm{top})[S]$ и $x \in S$

$$
((D, \preceq, g) \stackrel{\tau}{\rightarrow} x) \stackrel{\text { def }}{\Longleftrightarrow}\left(N_{\tau}(x) \subset(S \text {-ass })[D ; \preceq ; g]\right) .
$$

Если $(S, \tau)$ есть ТП и $\left(s_{i}\right)_{i \in \mathbb{N}}: \mathbb{N} \rightarrow S$, то обозначение в (3.1) упрощаем: при $x \in X$ факт сходимости $\left(s_{i}\right)_{i \in \mathbb{N}}$ к $x$ в $(S, \tau)$ записываем в виде $\left(s_{i}\right)_{i \in \mathbb{N}} \stackrel{\tau}{\rightarrow} x$.

Фиксируем до конца раздела непустые множества $X$ и $Y$, семейство $\mathcal{X} \in \mathcal{P}^{\prime}(\mathcal{P}(X))$, топологию $\tau \in(\mathrm{top})[Y]$ и отображение $r \in Y^{X}$. Следуя [11, определение 3.1], полагаем, что (as) $[X ; Y ; \tau ; r ; \mathcal{X}]$ есть по определению множество всех $y \in Y$, для каждого из которых существует такая направленность $(D, \preceq, g)$ в $X$, что

$$
(\mathcal{X} \subset(X \text {-ass })[D ; \preceq ; g]) \&((D, \preceq, r \circ g) \stackrel{\tau}{\rightarrow} y)
$$


(здесь и ниже о - символ композиции отображений). Если $\mathcal{X} \in \beta[X]$, то [17, (3.7)]

$$
\text { (as) }[X ; Y ; \tau ; r ; \mathcal{X}]=\bigcap_{B \in \mathcal{X}} \operatorname{cl}\left(r^{1}(B), \tau\right) .
$$

В связи с "секвенциальными" представлениями полагаем $\overrightarrow{m, \infty} \triangleq\{k \in \mathbb{N} \mid m \leq k\}$. Если $\mathcal{X} \in \mathcal{P}^{\prime}(\mathcal{P}(X))$, то $[17,(3.11)]$

$$
(\operatorname{sas})[X ; Y ; \tau ; r ; \mathcal{X}] \triangleq\left\{y \in Y \mid \exists\left(x_{i}\right)_{i \in \mathbb{N}} \in(\mathcal{X} \text {-seq })[X]:\left(r\left(x_{i}\right)\right)_{i \in \mathbb{N}} \stackrel{\tau}{\rightarrow} y\right\} \subset(\operatorname{as})[X ; Y ; \tau ; r ; \mathcal{X}],
$$

где (здесь и ниже)

$$
\left(\mathcal{X}_{\text {-seq })}[X] \triangleq\left\{\left(x_{i}\right)_{i \in \mathbb{N}} \in X^{\mathbb{N}} \mid \forall B \in \mathcal{X} \exists m \in \mathbb{N}: x_{k} \in B \forall k \in \overrightarrow{m, \infty}\right\} .\right.
$$

Достаточные условия совпадения множеств в (3.3) см. в [17, предложение 3.2$]$. В дальнейшем множество в правой части (3.3) именуем МП, а $(\operatorname{sas})[X ; Y ; \tau ; r ; \mathcal{X}]-$ секвенциальным МП.

Компактифицируемость. В связи с МП напомним [17, (4.1)], что если $E-$ непустое множество, $(Y, \tau)$ - хаусдорфово и $(\mathbf{K}, \mathbf{t})$ - компактное ТП, $p \in \mathbf{K}^{E}$ и $q \in C(\mathbf{K}, \mathbf{t}, Y, \tau)$, то

$$
\left(\text { as) }[E ; Y ; \tau ; q \circ p ; \mathcal{E}]=q^{1}((\operatorname{as})[E ; \mathbf{K} ; \mathbf{t} ; p ; \mathcal{E}]) \quad \forall \mathcal{E} \in \mathcal{P}^{\prime}(\mathcal{P}(E)) .\right.
$$

Далее рассматриваем (3.4) как основной способ исследования одних (более "сложных") МП в терминах других (более "простых").

\section{4. КОНЕЧНО АДДИТИВНЫЕ МЕРЫ И ЯРУСНЫЕ ФУНКЦИИ}

Всюду в дальнейшем фиксируем $a \in \mathbb{R}$ и $b \in \mathbb{R}$ со свойством $a<b$ ( $\mathbb{R}$ - вещественная прямая); рассматриваем $I \triangleq[a, b]$ в качестве промежутка управления, допуская, однако, и другие возможные интерпретации. Пусть

$$
\mathcal{I} \triangleq\{L \in \mathcal{P}(I) \mid \exists c, d \in I:(] c, d[\subset L) \&(L \subset[c, d])\} ;
$$

здесь и ниже для обозначения промежутков $\mathbb{R}$ используем только квадратные скобки (см. $[2$, гл. I; 10 , т. $1, \S 1.3])$. В (4.1) введена полуалгебра п/м $I$; через $\mathcal{A}$ обозначаем алгебру п/м $I$, порожденную полуалгеброй $\mathcal{I}(4.1): \mathcal{A}$ - семейство всех п/м $I$, допускающих каждое конечное разбиение множествами из $\mathcal{I}(4.1)$. Итак, $\mathcal{A} \in(\mathrm{alg})[I]$, а $(I, \mathcal{A})$ есть измеримое пространство с алгеброй множеств. Разумеется, $\mathcal{I} \subset \mathcal{A}$; конкретное представление $\mathcal{A}$ приведено в [10, т. 2 , (6.3.25)]. Для каждого множества $L \in \mathcal{P}(I)$ через $\chi_{L} \in \mathbb{R}^{I}$ обозначаем индикатор $L$ [33, II.2] (см. также [10, т. $1,(2.7 .1)]): \chi_{L}(x) \triangleq 1$ при $x \in L, \chi_{L}(y) \triangleq 0$ при $y \in I \backslash L$. Здесь и ниже мы используем обозначения [10]. Замыкание линейной оболочки $B_{0}(I, \mathcal{A})$ множества $\left\{\chi_{L}: L \in \mathcal{A}\right\}$ (в пространствах в/з функций линейные операции, умножение и порядок определяем поточечно) в банаховом пространстве $\mathbb{B}(I)$ (всех) ограниченных в/з функций на $I$ с топологией supнормы $\|\cdot\|($ см. $[24$, гл. IV,$\S 2 ; 10$, т. $1, \S 2.6])$ обозначаем через $B(I, \mathcal{A})$, что согласуется с $[24$, гл. IV]. Само $B(I, \mathcal{A})$ с нормой, индуцированной из $(\mathbb{B}(I),\|\cdot\|)$, есть банахово пространство, $B^{*}(I, \mathcal{A})$ - топологическое сопряженное к $B(I, \mathcal{A})$ - изометрически изоморфно пространству $\mathbb{A}(\mathcal{A})$ (всех) ограниченных в/з к.а. мер на $\mathcal{A}$ в оснащении (сильной) нормой-вариацией (см. [24, гл. IV], а также [10, т. 1 , теорема $3.6 .1,(4.11 .6)])$. Конкретный изометрический изоморфизм $\mathbb{A}(\mathcal{A})$ на $B^{*}(I, \mathcal{A})$ задается формулой

$$
\mu \mapsto\left(\int_{I} f d \mu\right)_{f \in B(I, \mathcal{A})}: \mathbb{A}(\mathcal{A}) \rightarrow B^{*}(I, \mathcal{A}),
$$


где операция интегрирования определяется простейшей схемой $[10$, т. 1 , гл. 3]. Тогда имеем двойственность топологических векторных пространств $(B(I, \mathcal{A}), \mathbb{A}(\mathcal{A}))$, а потому $\mathbb{A}(\mathcal{A})$ оснащаем обычной $*$-слабой топологией $\tau_{*}(\mathcal{A}) \in(\mathrm{top})[\mathbb{A}(\mathcal{A})] ;\left(\mathbb{A}(\mathcal{A}), \tau_{*}(\mathcal{A})\right)$ - локально выпуклый $\sigma$-компакт, в котором условия компактности определяются теоремой Алаоглу $[24$, гл. $\mathrm{V}, \S 4]$ (в конкретизированном виде см. $[6,(3.4 .19)])$. При этом $\mathbb{A}(\mathcal{A})$ порождается конусом $(\mathrm{add})_{+}[\mathcal{A}]$ (всех) неотрицательных в/з к.а. мер на $\mathcal{A}$. В терминах множества $\mathbb{P}(\mathcal{A}) \triangleq\left\{\mu \in(\mathrm{add})_{+}[\mathcal{A}] \mid\right.$ $\mu(I)=1\} \in\left(\tau_{*}(\mathcal{A})\right.$-comp $)[\mathbb{A}(\mathcal{A})]$ (всех к.а. вероятностей на $\left.\mathcal{A}\right)$ определяется множество

$$
\mathbb{T}(\mathcal{A}) \triangleq\{\mu \in \mathbb{P}(\mathcal{A}) \mid \forall A \in \mathcal{A}(\mu(A)=0) \vee(\mu(A)=1)\} \in\left(\tau_{*}(\mathcal{A}) \text {-comp }\right)[\mathbb{A}(\mathcal{A})],
$$

отождествимое с $\mathbb{F}_{0}^{*}(\mathcal{A})$. В самом деле, введем при $\mathcal{L} \in \mathcal{P}(\mathcal{A})$ индикатор $\mathcal{L}$, определенный на $\mathcal{A}$ : полагаем, что функция множеств $\mathbb{X}_{\mathcal{L}} \in \mathbb{R}^{\mathcal{A}}$ такова, что $\mathbb{X}_{\mathcal{L}}(L) \triangleq 1$ при $L \in \mathcal{L}$ и $\mathbb{X}_{\mathcal{L}}(A) \triangleq 0$ при $A \in \mathcal{A} \backslash \mathcal{L}$. Тогда $\mathbb{X}_{\mathcal{U}} \in \mathbb{T}(\mathcal{A})$ для всех $\mathcal{U} \in \mathbb{F}_{0}^{*}(\mathcal{A})$. Более того, отображение

$$
\varkappa \triangleq\left(\mathbb{X}_{\mathcal{U}}\right)_{\mathcal{U} \in \mathbb{F}_{0}^{*}(\mathcal{A})}
$$

есть биекция $\mathbb{F}_{0}^{*}(\mathcal{A})$ на $\mathbb{T}(\mathcal{A})$ (в $[19$, предложение 4.2$]$ это свойство усиливается до гомеоморфности при оснащении $\mathbb{F}_{0}^{*}(\mathcal{A})$ и $\mathbb{T}(\mathcal{A})$ некоторыми естественными топологиями); $\varkappa(\mathcal{U})=\mathbb{X}_{\mathcal{U}}$ для всех $\mathcal{U} \in \mathbb{F}_{0}^{*}(\mathcal{A})$. Напомним представление $\mathbb{F}_{0}^{*}(\mathcal{A})$, установленное в [13].

Если $t \in] a, b]$, то БФ $\mathcal{J}_{t}^{(-)} \triangleq\left\{\left[c, t\left[: c \in\left[a, t[\} \in \beta_{\mathcal{A}}^{00}[I]\right.\right.\right.\right.$ порождает (свободный) $\mathrm{y} / \phi \mathcal{U}_{t}^{(-)} \triangleq$ $\triangleq\left(I\right.$-fi) $\left[\mathcal{J}_{t}^{(-)} \mid \mathcal{A}\right] \in \mathbb{F}_{0}^{*}(\mathcal{A})$ с пустым пересечением всех своих множеств. Кроме того, при $t \in[a, b[$ БФ $\left.\left.\left.\left.\mathcal{J}_{t}^{(+)} \triangleq\{] t, c\right]: c \in\right] t, b\right]\right\} \in \beta_{\mathcal{A}}^{00}[I]$ порождает у $/ \phi \mathcal{U}_{t}^{(+)} \triangleq\left(I\right.$-fi) $\left[\mathcal{J}_{t}^{(+)} \mid \mathcal{A}\right] \in \mathbb{F}_{0}^{*}(\mathcal{A})$ с аналогичным свойством пустого пересечения. Тогда [13, разд. 6]

$$
\left.\left.\mathbb{F}_{0}^{*}(\mathcal{A})=\left\{\mathcal{U}_{t}^{(-)}: t \in\right] a, b\right]\right\} \cup\left\{\mathcal{U}_{t}^{(+)}: t \in[a, b[\} \cup\{((I, \mathcal{A}) \text {-ult })[t]: t \in I\} .\right.
$$

С учетом (4.2), (4.3) имеем $\varkappa\left(\mathcal{U}_{t}^{(-)}\right) \in \mathbb{T}(\mathcal{A})$ при $\left.\left.t \in\right] a, b\right]$ и $\varkappa\left(\mathcal{U}_{t}^{(+)}\right) \in \mathbb{T}(\mathcal{A})$ при $t \in[a, b[$.

Далее используются топологии $\tau_{\otimes}(\mathcal{A}) \in(\operatorname{top})[\mathbb{A}(\mathcal{A})]$ и $\tau_{0}(\mathcal{A}) \in(\operatorname{top})[\mathbb{A}(\mathcal{A})]$, определенные в $[5,(4.2 .8),(4.2 .9)]: \tau_{\otimes}(\mathcal{A})$ - топология поточечной сходимости в $\mathbb{A}(\mathcal{A})$, а $\tau_{0}(\mathcal{A})$ отвечает подпространству тихоновской степени $\mathbb{R}$ в дискретной топологии при условии, что $\mathcal{A}$ используется в качестве индексного множества.

Через $\eta$ обозначается след меры Лебега на алгебре $\mathcal{A}$; в частности, $\eta \in(\operatorname{add})_{+}[\mathcal{A}]$. В виде

$$
(\operatorname{add})^{+}[\mathcal{A} ; \eta] \triangleq\left\{\mu \in(\operatorname{add})_{+}[\mathcal{A}] \mid \forall A \in \mathcal{A}(\eta(A)=0) \Rightarrow(\mu(A)=0)\right\}
$$

имеем конус; посредством

$$
\begin{aligned}
& \mathbb{P}_{\eta}(\mathcal{A}) \triangleq \mathbb{P}(\mathcal{A}) \cap(\text { add })^{+}[\mathcal{A}, \eta] \in\left(\tau_{*}(\mathcal{A}) \text {-comp }\right)[\mathbb{A}(\mathcal{A})], \\
& \mathbb{T}_{\eta}(\mathcal{A}) \triangleq \mathbb{T}(\mathcal{A}) \cap(\text { add })^{+}[\mathcal{A}, \eta] \in\left(\tau_{*}(\mathcal{A}) \text {-comp }\right)[\mathbb{A}(\mathcal{A})]
\end{aligned}
$$

введены к.а. вероятности со свойством слабой абсолютной непрерывности [1]. Если $f \in B(I, \mathcal{A})$, то упомянутым свойством обладает (см. [10, т. $1, \S 3.7]$ )

$$
f * \eta \triangleq\left(\int_{L} f d \eta\right)_{L \in \mathcal{A}} \in \mathbb{A}(\mathcal{A})
$$

(неопределенный $\eta$-интеграл $f$ ). В терминах конуса $B_{0}^{+}(I, \mathcal{A}) \triangleq\left\{f \in B_{0}(I, \mathcal{A}) \mid f(t) \geq 0 \forall t \in I\right\}$ определяем непустое множество

$$
\mathbf{F} \triangleq\left\{\begin{array}{l|l}
f \in B_{0}^{+}(I, \mathcal{A}) \mid \int_{I} f d \eta=1
\end{array}\right\} \in \mathcal{P}^{\prime}\left(B_{0}^{+}(I, \mathcal{A})\right),
$$


элементы которого используются в качестве обычных (реализуемых) управлений; $f * \eta \in \mathbb{P}_{\eta}(\mathcal{A})$ для всех $f \in \mathbf{F}$. С учетом этого введем оператор $\mathfrak{I}$ посредством правила $f \mapsto f * \eta: \mathbf{F} \rightarrow \mathbb{P}_{\eta}(\mathcal{A})$; с учетом $[6$, Theorem 3.7 .2$]$ в виде $\mathfrak{I}^{1}(\mathbf{F})$ имеем всюду плотное п $/$ м $\mathbb{P}_{\eta}(\mathcal{A})$ :

$$
\mathbb{P}_{\eta}(\mathcal{A})=\operatorname{cl}\left(\mathfrak{I}^{1}(\mathbf{F}), \tau_{*}(\mathcal{A})\right)=\operatorname{cl}\left(\mathfrak{I}^{1}(\mathbf{F}), \tau_{\otimes}(\mathcal{A})\right)=\operatorname{cl}\left(\mathfrak{I}^{1}(\mathbf{F}), \tau_{0}(\mathcal{A})\right) .
$$

В частности (см. (4.4), (4.6)), имеем всюду плотное вложение $\mathbf{F}$ (4.5) в компакт. Напомним, что (см. [20])

$$
\left.\left.\mathbb{T}_{\eta}(\mathcal{A})=\mathbb{T}(\mathcal{A}) \cap \operatorname{cl}\left(\mathfrak{I}^{1}(\mathbf{F}), \tau_{*}(\mathcal{A})\right)=\left\{\varkappa\left(\mathcal{U}_{t}^{(-)}\right): t \in\right] a, b\right]\right\} \cup\left\{\varkappa\left(\mathcal{U}_{t}^{(+)}\right): t \in[a, b[\} .\right.
$$

\section{5. СОБЛЮДЕНИЕ ОГРАНИЧЕНИЙ В КЛАССЕ ОБОБЩЕННЫХ УПРАВЛЕНИЙ}

С учетом (4.6) мы будем рассматривать $\mathbb{P}_{\eta}(\mathcal{A})$ в качестве множества ОУ. В настоящем разделе будут введены ограничения на выбор $f \in \mathbf{F}$ и их обобщенные аналоги. Среди упомянутых ограничений будем выделять требование исчезающе малой продолжительности импульса и ограничения моментного характера; последние будем ослаблять различными способами. $\mathrm{B}$ результате в $\mathbb{P}_{\eta}(\mathcal{A})$ при оснащении различными топологиями будут возникать на уровне определений различные МП. Тем не менее удается установить определенную универсальность обобщенной задачи как способа представления упомянутых МП. Для реализации этой цели нам потребуются некоторые определения.

Если $t \in] a, b\left[\right.$, то при $\left.\zeta_{t}^{0} \triangleq \inf (\{t-a ; b-t\}) \in\right] 0, \infty[$ полагаем

$$
\left.\left.\left.\mathbb{P}_{\eta}^{0}(\mathcal{A} \mid t) \triangleq\left\{\mu \in \mathbb{P}_{\eta}(\mathcal{A}) \mid \mu(] t-\epsilon, t+\epsilon[)=1 \forall \epsilon \in\right] 0, \zeta_{t}^{0}\right]\right\} \quad \forall t \in\right] a, b[;
$$

легко видеть, что при $\mu \in \mathbb{P}_{\eta}^{0}(\mathcal{A} \mid t)$ непременно $\mu=\mu\left(\left[a, t[) \varkappa\left(\mathcal{U}_{t}^{(-)}\right)+\mu(] t, b\right]\right) \varkappa\left(\mathcal{U}_{t}^{(+)}\right)$. Более того,

$$
\left.\mathbb{P}_{\eta}^{0}(\mathcal{A} \mid t)=\left\{\alpha \varkappa\left(\mathcal{U}_{t}^{(-)}\right)+(1-\alpha) \varkappa\left(\mathcal{U}_{t}^{(+)}\right): \alpha \in[0,1]\right\} \quad \forall t \in\right] a, b[.
$$

В терминах множеств (5.1) далее конструируем множество ОУ, “допустимых" в смысле исчезающе малой продолжительности импульса (см. [22, теорема 4.1]). Пусть

$$
\mathbb{P}_{\eta}^{0}[\mathcal{A}] \triangleq\left(\bigcup_{t \in] a, b[} \mathbb{P}_{\eta}^{0}(\mathcal{A} \mid t)\right) \cup\left\{\varkappa\left(\mathcal{U}_{a}^{(+)}\right) ; \varkappa\left(\mathcal{U}_{b}^{(-)}\right)\right\} \in \mathcal{P}^{\prime}\left(\mathbb{P}_{\eta}(\mathcal{A})\right) .
$$

Ограничения моментного характера. Фиксируем $N \in \mathbb{N}$ и кортеж $\left(\rho_{i}\right)_{i \in \overline{1, N}}: \overline{1, N} \rightarrow$ $\rightarrow B(I, \mathcal{A})$. Итак,

$$
\rho_{i} \in B(I, \mathcal{A}) \quad \forall i \in \overline{1, N}
$$

Через $\tau_{\mathbb{R}}^{(N)}$ обозначаем обычную нормируемую топологию покоординатной сходимости в $\mathbb{R}^{N}$ и фиксируем (непустое) множество $\mathbf{Y} \in \mathcal{P}^{\prime}\left(\mathbb{R}^{N}\right)$, замкнутое в $\left(\mathbb{R}^{N}, \tau_{\mathbb{R}}^{(N)}\right)$. Ниже рассматривается следующее условие на выбор $f \in \mathbf{F}$ :

$$
\left(\int_{I} \rho_{i} f d \eta\right)_{i \in \overline{1, N}} \in \mathbf{Y}
$$

(ограничение моментного характера). Мы рассмотрим два варианта ослабления (5.4). Полагаем сначала, что при $\varepsilon \in] 0, \infty[$

$$
O(\mathbf{Y}, \varepsilon) \triangleq\left\{\left(z_{i}\right)_{i \in \overline{1, N}} \in \mathbb{R}^{N}\left|\exists\left(y_{i}\right)_{i \in \overline{1, N}} \in \mathbf{Y}:\right| y_{j}-z_{j} \mid<\varepsilon \forall j \in \overline{1, N}\right\},
$$


получая обычную "равномерную" покоординатно $\varepsilon$-окрестность $\mathbf{Y}$. Кроме того, фиксируем $M \in \mathcal{P}(\overline{1, N})$ со свойством

$$
\rho_{j} \in B_{0}(I, \mathcal{A}) \quad \forall j \in M
$$

в виде $M, M \subset \overline{1, N}$, имеем "множество ступенчатости" для набора функций (5.3). При $\varepsilon \in] 0, \infty[$ полагаем

$$
\widehat{O}(\mathbf{Y}, \varepsilon) \triangleq\left\{\left(z_{i}\right)_{i \in \overline{1, N}} \in \mathbb{R}^{N} \mid \exists\left(y_{i}\right)_{i \in \overline{1, N}} \in \mathbf{Y}:\left(y_{j}=z_{j} \forall j \in M\right) \&\left(\left|y_{j}-z_{j}\right|<\varepsilon \forall j \in \overline{1, N}\right)\right\},
$$

получая "неравномерную" в покоординатном смысле $\varepsilon$-окрестность Ү. Из (5.5) и (5.6) следует, что $\widehat{O}(\mathbf{Y}, \varepsilon) \subset O(\mathbf{Y}, \varepsilon)$ при $\varepsilon \in] 0, \infty[$. Отметим, что условия типа $(5.5)$ использовались в $[5-7,9$, 23] и других работах в связи с исследованием свойства асимптотической нечувствительности при ослаблении части ограничений.

Возвращаясь к (4.5), напомним некоторые определения из [22]. Если $f \in \mathbf{F}$, то $\operatorname{supp}(f) \triangleq$ $\triangleq\{t \in I \mid f(t) \neq 0\} \in \mathcal{P}^{\prime}(I)$ и мы получаем $\mathbf{t}_{0}(f) \triangleq \inf (\operatorname{supp}(f)) \in I, \mathbf{t}^{0}(f) \triangleq \sup (\operatorname{supp}(f)) \in I$ и

$$
\mathbf{t}_{f} \triangleq \frac{\mathbf{t}_{0}(f)+\mathbf{t}^{0}(f)}{2} \in I
$$

$a \leq \mathbf{t}_{0}(f) \leq \mathbf{t}_{f} \leq \mathbf{t}^{0}(f) \leq b$. С учетом этого, следуя [22], введем при $\left.\varepsilon \in\right] 0, \infty[$ множество $\mathbf{F}_{\varepsilon} \triangleq\left\{f \in \mathbf{F} \mid \mathbf{t}^{0}(f)-\mathbf{t}_{0}(f)<\varepsilon\right\}$, а также

$$
\begin{aligned}
& \mathbb{Y}_{\varepsilon} \triangleq\left\{f \in \mathbf{F}_{\varepsilon} \mid\left(\int_{I} \rho_{i} f d \eta\right)_{i \in \overline{1, N}} \in O(\mathbf{Y}, \varepsilon)\right\}, \\
& \widehat{\mathbb{Y}}_{\varepsilon} \triangleq\left\{f \in \mathbf{F}_{\varepsilon} \mid\left(\int_{I} \rho_{i} f d \eta\right)_{i \in \overline{1, N}} \in \widehat{O}(\mathbf{Y}, \varepsilon)\right\} .
\end{aligned}
$$

Полагаем далее $\mathfrak{Y} \triangleq\left\{\mathbb{Y}_{\varepsilon}: \varepsilon \in\right] 0, \infty[\}$ и $\widehat{\mathfrak{Y}} \triangleq\left\{\widehat{\mathbb{Y}}_{\varepsilon}: \varepsilon \in\right] 0, \infty[\}$, получая два варианта ОАХ: $\mathfrak{Y} \in \beta[\mathbf{F}]$ и $\widehat{\mathfrak{Y}} \in \beta[\mathbf{F}]$. Поэтому при $\tau \in(\mathrm{top})\left[\mathbb{P}_{\eta}(\mathcal{A})\right]$ определены МП в $\left(\mathbb{P}_{\eta}(\mathcal{A}), \tau\right)$ на значениях $\mathfrak{I}$, отвечающие ОАХ для направленных семейств $\mathfrak{Y}$ и $\widehat{\mathfrak{Y}}$. В интересах представления упомянутых МП введем множество

$$
\widetilde{\mathbb{P}}_{\eta}^{0}(\mathcal{A}) \triangleq\left\{\mu \in \mathbb{P}_{\eta}^{0}[\mathcal{A}] \mid\left(\int_{I} \rho_{i} d \mu\right)_{i \in \overline{1, N}} \in \mathbf{Y}\right\}
$$

элементы (5.8) играют роль допустимых ОУ. Отметим теперь, что используемые топологии множества $\mathbb{P}_{\eta}(\mathcal{A})$ сравнимы (см. [5, Sect. 4.2]):

$$
\left.\tau_{\eta}^{*}(\mathcal{A}) \triangleq \tau_{*}(\mathcal{A})\right|_{\mathbb{P}_{\eta}(\mathcal{A})}=\left.\tau_{\otimes}(\mathcal{A})\right|_{\mathbb{P}_{\eta}(\mathcal{A})} \subset \tau_{\eta}^{0}(\mathcal{A})
$$

где (здесь и ниже) $\left.\tau_{\eta}^{0}(\mathcal{A}) \triangleq \tau_{0}(\mathcal{A})\right|_{\mathbb{P}_{\eta}(\mathcal{A})}$. Вполне очевидна (см. $\left.(5.9)\right)$ следующая

Лемма 5.1. Если $\mathcal{S} \in \beta[\mathbf{F}]$, то $(\operatorname{as})\left[\mathbf{F} ; \mathbb{P}_{\eta}(\mathcal{A}) ; \tau_{\eta}^{*}(\mathcal{A}) ; \mathfrak{I} ; \mathcal{S}\right]$ есть множество всех к.а. мер $\mu \in \mathbb{P}_{\eta}(\mathcal{A})$, для каждой из которых существует такая направленность $(D, \preceq, g)$ в $\mathbf{F}$, что

$$
(\mathcal{S} \subset(\mathbf{F} \text {-ass })[D ; \preceq ; g]) \&\left((D, \preceq, \mathfrak{I} \circ g) \stackrel{\tau_{\otimes}(\mathcal{A})}{\longrightarrow} \mu\right) .
$$

Предложение 5.1. Множество (5.8) допускает следующую оценку снизу:

$$
\text { (as) }\left[\mathbf{F} ; \mathbb{P}_{\eta}(\mathcal{A}) ; \tau_{\eta}^{*}(\mathcal{A}) ; \mathfrak{I} ; \mathfrak{Y}\right] \subset \widetilde{\mathbb{P}}_{\eta}^{0}(\mathcal{A}) .
$$


Доказательство. Выберем произвольную к.а. меру $\mu_{0}$ из множества в левой части (5.10). Тогда $\mu_{0} \in \mathbb{P}_{\eta}(\mathcal{A})$ и (см. лемму 5.1) для некоторой направленности $(D, \preceq, g)$ в $\mathbf{F}$

$$
(\mathfrak{Y} \subset(\mathbf{F} \text {-ass })[D ; \preceq ; g]) \&\left((D, \preceq, \mathfrak{I} \circ g) \stackrel{\tau_{\otimes}(\mathcal{A})}{\longrightarrow} \mu_{0}\right) .
$$

Кроме того, $(D, \preceq, \mathfrak{I} \circ g) \stackrel{\tau_{*}(\mathcal{A})}{\longrightarrow} \mu_{0}$ (см. разд. 3 , и в частности $\left.(3.2)\right)$. Здесь $(D, \preceq)-$ непустое направленное множество $(\mathrm{HM}), g \in \mathbf{F}^{D}$. Тогда $\left(D, \preceq, \mathbf{t}_{g(\cdot)}\right)$, где $\mathbf{t}_{g(\cdot)} \triangleq\left(\mathbf{t}_{g(d)}\right)_{d \in D} \in I^{D}$, есть направленность в $I$. С учетом компактности $I$ в обычной топологии, порождаемой метрикой $(x, y) \mapsto|x-y|: I \times I \rightarrow\left[0, \infty\left[\right.\right.$, получаем возможность изотонного прореживания $\left(D, \preceq, \mathbf{t}_{g(\cdot)}\right)$ до сходящейся поднаправленности (см. $[23,(2.3 .23)])$. В этой связи полагаем, что для каждого непустого $\mathrm{HM}(\mathcal{D}, \propto)$

$$
\begin{aligned}
(\text { Isot })[\mathcal{D}, \propto ; D, \preceq] \triangleq & \left\{h \in D^{\mathcal{D}} \mid\left(h^{1}(\mathcal{D}) \in(\preceq \text {-cof })[D]\right) \&\right. \\
& \left.\&\left(\forall d_{1} \in \mathcal{D} \forall d_{2} \in \mathcal{D}\left(d_{1} \propto d_{2}\right) \Rightarrow\left(h\left(d_{1}\right) \preceq h\left(d_{2}\right)\right)\right)\right\},
\end{aligned}
$$

где $(\preceq$-cof $)[D] \triangleq\left\{H \in \mathcal{P}(D) \mid \forall d^{\prime} \in D \exists d^{\prime \prime} \in H: d^{\prime} \preceq d^{\prime \prime}\right\}$. В силу упомянутой выше компактности $I$ найдутся такие $\mathrm{HM}(\mathbb{D}, \sqsubseteq)$, отображение $\lambda \in(\mathrm{Isot})[\mathbb{D}, \sqsubseteq ; D, \preceq]$ и число $t_{0} \in I$, что

$$
\forall \varepsilon \in] 0, \infty\left[\quad \exists d^{\prime} \in \mathbb{D} \quad \forall d^{\prime \prime} \in \mathbb{D} \quad\left(d^{\prime} \sqsubseteq d^{\prime \prime}\right) \Rightarrow\left(\left|\mathbf{t}_{g\left(\lambda\left(d^{\prime \prime}\right)\right)}-t_{0}\right|<\varepsilon\right) .\right.
$$

Из (5.11) по свойствам $\lambda$ имеем сходимость $(\mathbb{D}, \sqsubseteq, \mathfrak{I} \circ g \circ \lambda) \stackrel{\tau_{\otimes}(\mathcal{A})}{\longrightarrow} \mu_{0}$. С другой стороны, из $(5.9)$ следует (см. (3.2)), что [23, (2.3.9)]

$$
(\mathbb{D}, \sqsubseteq, \mathfrak{I} \circ g \circ \lambda) \stackrel{\tau_{*}(\mathcal{A})}{\longrightarrow} \mu_{0} .
$$

Кроме того, из (5.11) по свойствам $\lambda$ имеем

$$
\mathfrak{Y} \subset(\mathbf{F} \text {-ass })[\mathbb{D} ; \sqsubseteq ; g \circ \lambda] .
$$

Пусть $\widehat{\xi} \in] 0, \infty\left[\right.$ и $\widetilde{\xi} \triangleq \widehat{\xi} / 2$. Из (5.13) вытекает, что $\mathbb{Y}_{\widetilde{\xi}} \in(\mathbf{F}$-ass $)[\mathbb{D} ; \sqsubseteq ; g \circ \lambda]$, а тогда для некоторого $d_{0} \in \mathbb{D}$ имеем

$$
\left(d_{0} \sqsubseteq \delta\right) \Rightarrow\left(\left(\int_{I} \rho_{i} d(\mathfrak{I} \circ g \circ \lambda)(\delta)\right)_{i \in \overline{1, N}} \in O(\mathbf{Y}, \widetilde{\xi})\right) \quad \forall \delta \in \mathbb{D} .
$$

С учетом (5.12) имеем, однако, для некоторого $d^{*} \in \mathbb{D}$ свойство

$$
\left(d^{*} \sqsubseteq \delta\right) \Rightarrow\left(\left|\int_{I} \rho_{i} d(\mathfrak{I} \circ g \circ \lambda)(\delta)-\int_{I} \rho_{i} d \mu_{0}\right|<\widetilde{\xi} \quad \forall i \in \overline{1, N}\right) \quad \forall \delta \in \mathbb{D} .
$$

Из (5.6), (5.14), (5.15) по свойствам НМ получаем $\left(\int_{I} \rho_{i} d \mu_{0}\right)_{i \in \overline{1, N}} \in O(\mathbf{Y}, \widehat{\xi})$. Поскольку выбор $\widehat{\xi}$ был произвольным, в силу замкнутости $\mathbf{Y}$ имеем свойство

$$
\left(\int_{I} \rho_{i} d \mu_{0}\right)_{i \in \overline{1, N}} \in \mathbf{Y}
$$

Далее, подобно аналогичному рассуждению в [22] проверяется, что $\left(\left(t_{0}=a\right) \Rightarrow\left(\mu_{0}=\varkappa\left(\mathcal{U}_{a}^{(+)}\right)\right)\right) \&\left(\left(t_{0} \in\right] a, b[) \Rightarrow\left(\mu_{0} \in \mathbb{P}_{\eta}^{0}\left(\mathcal{A} \mid t_{0}\right)\right)\right) \&\left(\left(t_{0}=b\right) \Rightarrow\left(\mu_{0}=\varkappa\left(\mathcal{U}_{b}^{(-)}\right)\right)\right)$. 
Как следствие, $\mu_{0} \in \mathbb{P}_{\eta}^{0}[\mathcal{A}]$, а потому с учетом (5.8), (5.16) имеем $\mu_{0} \in \widetilde{\mathbb{P}}_{\eta}^{0}(\mathcal{A})$, чем завершается доказательство (5.10).

С учетом (5.9) легко проверяются свойства

$$
\begin{aligned}
& (\mathrm{sas})\left[\mathbf{F} ; \mathbb{P}_{\eta}(\mathcal{A}) ; \tau_{\eta}^{0}(\mathcal{A}) ; \mathfrak{I} ; \mathfrak{Y}\right] \subset(\operatorname{sas})\left[\mathbf{F} ; \mathbb{P}_{\eta}(\mathcal{A}) ; \tau_{\eta}^{*}(\mathcal{A}) ; \mathfrak{I} ; \mathfrak{Y}\right] \\
& (\operatorname{sas})\left[\mathbf{F} ; \mathbb{P}_{\eta}(\mathcal{A}) ; \tau_{\eta}^{0}(\mathcal{A}) ; \mathfrak{I} ; \widehat{\mathfrak{Y}}\right] \subset(\operatorname{sas})\left[\mathbf{F} ; \mathbb{P}_{\eta}(\mathcal{A}) ; \tau_{\eta}^{*}(\mathcal{A}) ; \mathfrak{I} ; \widehat{\mathfrak{Y}}\right]
\end{aligned}
$$

Отметим также (см. (5.7)) очевидное следствие определений семейств $\mathfrak{Y}$ и $\widehat{\mathfrak{Y}}$ :

$$
(\operatorname{sas})\left[\mathbf{F} ; \mathbb{P}_{\eta}(\mathcal{A}) ; \tau ; \mathfrak{I} ; \widehat{\mathfrak{Y}}\right] \subset(\operatorname{sas})\left[\mathbf{F} ; \mathbb{P}_{\eta}(\mathcal{A}) ; \tau ; \mathfrak{I} ; \mathfrak{Y}\right] \quad \forall \tau \in(\text { top })\left[\mathbb{P}_{\eta}(\mathcal{A})\right]
$$

В связи с (5.17), (5.18) рассмотрим некоторые варианты секвенциальной реализации элементов вспомогательных МП. Если $t \in] a, b]$, то определено отображение

$$
\mathfrak{X}_{t}^{(-)} \triangleq\left(\frac{1}{t-c} \chi_{[c, t[}\right)_{c \in[a, t[} \in \mathbf{F}^{[a, t]}
$$

Из определения БФ $\mathcal{J}_{t}^{(-)}$легко следует положение о секвенциальной реализации $\varkappa\left(\mathcal{U}_{t}^{(-)}\right)$. Именно, при $t \in] a, b]$ и $\left(c_{i}\right)_{i \in \mathbb{N}} \in\left[a, t\left[^{\mathbb{N}}\right.\right.$ истинна импликация

$$
\left(\left(c_{i}\right)_{i \in \mathbb{N}} \rightarrow t\right) \Rightarrow\left(\left(\Im\left(\mathfrak{X}_{t}^{(-)}\left(c_{i}\right)\right)\right)_{i \in \mathbb{N}} \stackrel{\tau_{\eta}^{0}(\mathcal{A})}{\longrightarrow} \varkappa\left(\mathcal{U}_{t}^{(-)}\right)\right) .
$$

Кроме того, из $(5.7),(5.19)$ и определения топологий $\tau_{\eta}^{*}(\mathcal{A}), \tau_{\eta}^{0}(\mathcal{A})$ вытекает следующее свойство: если $t \in] a, b], \varkappa\left(\mathcal{U}_{t}^{(-)}\right) \in \widetilde{\mathbb{P}}_{\eta}^{0}(\mathcal{A})$ и $\left(c_{i}\right)_{i \in \mathbb{N}} \in\left[a, t\left[^{\mathbb{N}}\right.\right.$, то

$$
\left(\left(c_{i}\right)_{i \in \mathbb{N}} \rightarrow t\right) \Rightarrow\left(\left(\mathfrak{X}_{t}^{(-)}\left(c_{i}\right)\right)_{i \in \mathbb{N}} \in(\widehat{\mathfrak{Y}} \text {-seq })[\mathbf{F}]\right) .
$$

Используя [13, (6.19)] и [10, т. 2, теорема 10.8.1], получаем

$$
\forall f \in B(I, \mathcal{A}) \quad \forall t \in] a, b] \quad \forall \varepsilon \in] 0, \infty\left[\exists c \in \left[a, t\left[: \quad\left|f(\zeta)-\int_{I} f d \varkappa\left(\mathcal{U}_{t}^{(-)}\right)\right|<\varepsilon \quad \forall \zeta \in[c, t[.\right.\right.\right.
$$

Данное свойство характеризует интеграл в (5.21) как предел слева соответствующей подынтегральной функции (см. также [16]): если $f \in B(I, \mathcal{A})$ и $t \in] a, b]$, то

$$
\int_{I} f d \varkappa\left(\mathcal{U}_{t}^{(-)}\right)=\lim _{\theta \uparrow t} f(\theta)
$$

В этой связи при $t \in] a, b]$ полагаем (см. (5.3), (5.22)) $\widehat{\rho}_{\uparrow}(t) \triangleq\left(\lim _{\theta \uparrow t} \rho_{i}(\theta)\right)_{i \in \overline{1, N}}$; тогда (см. (5.22))

$$
\left(\int_{I} \rho_{i} d \varkappa\left(\mathcal{U}_{t}^{(-)}\right)\right)_{i \in \overline{1, N}}=\widehat{\rho}_{\uparrow}(t) .
$$

Предложение 5.2. Если $t \in] a, b] u \widehat{\rho}_{\uparrow}(t) \in \mathbf{Y}, \operatorname{mo} \varkappa\left(\mathcal{U}_{t}^{(-)}\right) \in(\operatorname{sas})\left[\mathbf{F} ; \mathbb{P}_{\eta}(\mathcal{A}) ; \tau_{\eta}^{0}(\mathcal{A}) ; \mathfrak{I} ; \widehat{\mathfrak{Y}}\right]$. Доказательство получается непосредственной комбинацией (3.3), (5.19), (5.20) и (5.23). Отметим очевидное следствие:

$$
\left(\widehat{\rho} \uparrow_{\uparrow}(b) \in \mathbf{Y}\right) \Rightarrow\left(\varkappa\left(\mathcal{U}_{b}^{(-)}\right) \in(\operatorname{sas})\left[\mathbf{F} ; \mathbb{P}_{\eta}(\mathcal{A}) ; \tau_{\eta}^{0}(\mathcal{A}) ; \mathfrak{I} ; \widehat{\mathfrak{Y}}\right]\right)
$$


Если $t \in[a, b[$, определяем отображение

$$
\mathfrak{X}_{t}^{(+)} \triangleq\left(\frac{1}{c-t} \chi_{] t, c]}\right)_{c \in] t, b]} \in \mathbf{F}^{[t, b]}
$$

По аналогии с (5.19) устанавливается следующее весьма очевидное свойство: если $t \in[a, b[$ и $\left.\left.\left(c_{i}\right)_{i \in \mathbb{N}} \in\right] t, b\right]^{\mathbb{N}}$, то

$$
\left(\left(c_{i}\right)_{i \in \mathbb{N}} \rightarrow t\right) \Rightarrow\left(\left(\Im\left(\mathfrak{X}_{t}^{(+)}\left(c_{i}\right)\right)\right)_{i \in \mathbb{N}} \stackrel{\tau_{\eta}^{0}(\mathcal{A})}{\longrightarrow} \varkappa\left(\mathcal{U}_{t}^{(+)}\right)\right) .
$$

Далее, из $(5.7),(5.19)$ и определения топологий $\tau_{\eta}^{*}(\mathcal{A}), \tau_{\eta}^{0}(\mathcal{A})$ вытекает следующее свойство: если $t \in\left[a, b\left[, \varkappa\left(\mathcal{U}_{t}^{(+)}\right) \in \widetilde{\mathbb{P}}_{\eta}^{0}(\mathcal{A}) \text { и }\left(c_{i}\right)_{i \in \mathbb{N}} \in\right] t, b\right]^{\mathbb{N}}$, то

$$
\left(\left(c_{i}\right)_{i \in \mathbb{N}} \rightarrow t\right) \Rightarrow\left(\left(\mathfrak{X}_{t}^{(+)}\left(c_{i}\right)\right)_{i \in \mathbb{N}} \in(\widehat{\mathfrak{Y}} \text {-seq })[\mathbf{F}]\right) .
$$

Используя теперь $[13,(6.19)]$ и [10, т. 2, теорема 10.8.1], получаем

$$
\left.\left.\forall f \in B(I, \mathcal{A}) \quad \forall t \in[a, b[\forall \varepsilon \in] 0, \infty[\exists c \in] t, b]: \quad\left|f(\zeta)-\int_{I} f d \varkappa\left(\mathcal{U}_{t}^{(+)}\right)\right|<\varepsilon \quad \forall \zeta \in\right] t, c\right] .
$$

Данное свойство характеризует интеграл в (5.27) как предел справа соответствующей подынтегральной функции: если $f \in B(I, \mathcal{A})$ и $t \in[a, b[$, то

$$
\int_{I} f d \varkappa\left(\mathcal{U}_{t}^{(+)}\right)=\lim _{\theta \downarrow t} f(\theta) .
$$

В этой связи при $t \in\left[a, b\left[\right.\right.$ полагаем $\widehat{\rho}_{\downarrow}(t) \triangleq\left(\lim _{\theta \downarrow t} \rho_{i}(\theta)\right)_{i \in \overline{1, N}}$; тогда (см. (5.28))

$$
\left(\int_{I} \rho_{i} d \varkappa\left(\mathcal{U}_{t}^{(+)}\right)\right)_{i \in \overline{1, N}}=\widehat{\rho}_{\downarrow}(t)
$$

Предложение 5.3. Если $t \in\left[a, b\left[u \widehat{\rho}_{\downarrow}(t) \in \mathbf{Y}, \operatorname{mo} \varkappa\left(\mathcal{U}_{t}^{(+)}\right) \in(\operatorname{sas})\left[\mathbf{F} ; \mathbb{P}_{\eta}(\mathcal{A}) ; \tau_{\eta}^{0}(\mathcal{A}) ; \mathfrak{I} ; \widehat{\mathfrak{Y}}\right]\right.\right.$. Доказательство получается непосредственной комбинацией (3.3), (5.25), (5.26) и (5.29). Отметим полезное следствие:

$$
\left(\widehat{\rho}_{\downarrow}(a) \in \mathbf{Y}\right) \Rightarrow\left(\varkappa\left(\mathcal{U}_{a}^{(+)}\right) \in(\operatorname{sas})\left[\mathbf{F} ; \mathbb{P}_{\eta}(\mathcal{A}) ; \tau_{\eta}^{0}(\mathcal{A}) ; \mathfrak{I} ; \widehat{\mathfrak{Y}}\right]\right)
$$

Предложение 5.4. Если $t \in] a, b\left[,\left(c_{i}\right)_{i \in \mathbb{N}} \in\left[a, t\left[^{\mathbb{N}} u\left(\widetilde{c}_{i}\right)_{i \in \mathbb{N}} \in\right] t, b\right]^{\mathbb{N}}\right.$, mo

$$
\begin{aligned}
& \left(\left(\left(c_{i}\right)_{i \in \mathbb{N}} \rightarrow t\right) \&\left(\left(\widetilde{c}_{i}\right)_{i \in \mathbb{N}} \rightarrow t\right)\right) \Rightarrow \\
& \quad \Rightarrow\left(\left(\Im\left(\alpha \mathfrak{X}_{t}^{(-)}\left(c_{i}\right)+(1-\alpha) \mathfrak{X}_{t}^{(+)}\left(\widetilde{c}_{i}\right)\right)\right)_{i \in \mathbb{N}} \stackrel{\tau_{\eta}^{0}(\mathcal{A})}{\longrightarrow} \alpha \varkappa\left(\mathcal{U}_{t}^{(-)}\right)+(1-\alpha) \varkappa\left(\mathcal{U}_{t}^{(+)}\right) \forall \alpha \in[0,1]\right) .
\end{aligned}
$$

Доказательство следует из (5.20), (5.25) с учетом простейших свойств неопределенного $\eta$-интеграла.

В развитие предложений 5.2 и 5.3 отметим полезное следствие. 
Следствие 5.1. Если $t \in] a, b[, \alpha \in[0,1]$,

$$
\alpha \varkappa\left(\mathcal{U}_{t}^{(-)}\right)+(1-\alpha) \varkappa\left(\mathcal{U}_{t}^{(+)}\right) \in \widetilde{\mathbb{P}}_{\eta}^{0}(\mathcal{A}),
$$

$\left(c_{i}\right)_{i \in \mathbb{N}} \in\left[a, t\left[^{\mathbb{N}},\left(\widetilde{c}_{i}\right)_{i \in \mathbb{N}} \in\right] t, b\right]^{\mathbb{N}}$ и при этом

$$
\left(\left(c_{i}\right)_{i \in \mathbb{N}} \rightarrow t\right) \&\left(\left(\widetilde{c}_{i}\right)_{i \in \mathbb{N}} \rightarrow t\right)
$$

$m o\left(\alpha \mathfrak{X}_{t}^{(-)}\left(c_{i}\right)+(1-\alpha) \mathfrak{X}_{t}^{(+)}\left(\widetilde{c}_{i}\right)\right)_{i \in \mathbb{N}} \in(\widehat{\mathfrak{Y}}-$ seq $)[\mathbf{F}]$.

Доказательство использует $(5.8)$ и конструкции, вытекающие из определения $\tau_{\eta}^{0}(\mathcal{A})$ и подобные (5.20), (5.26). Упомянутые конструкции являются достаточно простыми; по этой причине весьма очевидное обоснование следствия опустим, отметив только представление $\tau_{\eta}^{0}(\mathcal{A})$, связанное с $[5,(4.2 .14)]$. Учитываем также (5.9).

Предложение 5.5. Если $t \in] a, b[$, mo

$$
\left\{\mu \in \mathbb{P}_{\eta}^{0}(\mathcal{A} \mid t) \mid\left(\int_{I} \rho_{i} d \mu\right)_{i \in \overline{1, N}} \in \mathbf{Y}\right\} \subset(\operatorname{sas})\left[\mathbf{F} ; \mathbb{P}_{\eta}(\mathcal{A}) ; \tau_{\eta}^{0}(\mathcal{A}) ; \mathfrak{I} ; \widehat{\mathfrak{Y}}\right] .
$$

Доказательство. Выберем произвольную к.а. меру $\mu_{0}$ из множества в левой части (5.32). Тогда $\mu_{0} \in \mathbb{P}_{\eta}^{0}(\mathcal{A} \mid t)$ и

$$
\left(\int_{I} \rho_{i} d \mu_{0}\right)_{i \in \overline{1, N}} \in \mathbf{Y}
$$

Поскольку $\mu_{0} \in \mathbb{P}_{\eta}^{0}[\mathcal{A}]$ согласно $(5.2)$, в силу (5.8) и (5.33) имеем $\mu_{0} \in \widetilde{\mathbb{P}}_{\eta}^{0}(\mathcal{A})$. С другой стороны, согласно (5.1)

$$
\mu_{0}=\alpha_{0} \varkappa\left(\mathcal{U}_{t}^{(-)}\right)+\left(1-\alpha_{0}\right) \varkappa\left(\mathcal{U}_{t}^{(+)}\right)
$$

где $\alpha_{0} \in[0,1]$. Теперь при $k \in \mathbb{N}$ полагаем

$$
c_{k} \triangleq t-\frac{t-a}{k}, \quad \widetilde{c}_{k} \triangleq t+\frac{b-t}{k} .
$$

Получаем последовательности $\left(c_{i}\right)_{i \in \mathbb{N}} \in\left[a, t\left[^{\mathbb{N}},\left(\widetilde{c}_{i}\right)_{i \in \mathbb{N}} \in\right] t, b\right]^{\mathbb{N}}$ со свойством (5.31). Теперь из (5.34) и следствия 5.1 для $f_{j}^{*} \triangleq \alpha_{0} \mathfrak{X}_{t}^{(-)}\left(c_{j}\right)+\left(1-\alpha_{0}\right) \mathfrak{X}_{t}^{(+)}\left(\widetilde{c}_{j}\right), j \in \mathbb{N}$, получаем свойство $\left(f_{k}^{*}\right)_{k \in \mathbb{N}} \in(\widehat{\mathfrak{Y}}$-seq) $[\mathbf{F}]$. При этом из предложения 5.4 и (5.34) вытекает сходимость

$$
\left(\mathfrak{I}\left(f_{k}^{*}\right)\right)_{k \in \mathbb{N}} \stackrel{\tau_{\eta}^{0}(\mathcal{A})}{\longrightarrow} \mu_{0} .
$$

С учетом $(3.3)$ получаем $\mu_{0} \in(\mathrm{sas})\left[\mathbf{F} ; \mathbb{P}_{\eta}(\mathcal{A}) ; \tau_{\eta}^{0}(\mathcal{A}) ; \mathfrak{I} ; \widehat{\mathfrak{Y}}\right]$. Итак, $(5.32)$ установлено.

Используя (5.8), (5.23), (5.24), (5.29), (5.30) и предложение 5.5 получаем

$$
\widetilde{\mathbb{P}}_{\eta}^{0}(\mathcal{A}) \subset(\operatorname{sas})\left[\mathbf{F} ; \mathbb{P}_{\eta}(\mathcal{A}) ; \tau_{\eta}^{0}(\mathcal{A}) ; \mathfrak{I} ; \widehat{\mathfrak{Y}}\right] .
$$

Теорема 5.1. Справедлива чепочка равенств

$$
\begin{aligned}
\widetilde{\mathbb{P}}_{\eta}^{0}(\mathcal{A}) & =(\operatorname{as})\left[\mathbf{F} ; \mathbb{P}_{\eta}(\mathcal{A}) ; \tau_{\eta}^{*}(\mathcal{A}) ; \mathfrak{I} ; \mathfrak{Y}\right]=(\operatorname{as})\left[\mathbf{F} ; \mathbb{P}_{\eta}(\mathcal{A}) ; \tau_{\eta}^{0}(\mathcal{A}) ; \mathfrak{I} ; \mathfrak{Y}\right]=(\operatorname{as})\left[\mathbf{F} ; \mathbb{P}_{\eta}(\mathcal{A}) ; \tau_{\eta}^{*}(\mathcal{A}) ; \mathfrak{I} ; \widehat{\mathfrak{Y}}\right]= \\
& =(\operatorname{as})\left[\mathbf{F} ; \mathbb{P}_{\eta}(\mathcal{A}) ; \tau_{\eta}^{0}(\mathcal{A}) ; \mathfrak{I} ; \widehat{\mathfrak{Y}}\right]=(\operatorname{sas})\left[\mathbf{F} ; \mathbb{P}_{\eta}(\mathcal{A}) ; \tau_{\eta}^{0}(\mathcal{A}) ; \mathfrak{I} ; \mathfrak{Y}\right]=(\operatorname{sas})\left[\mathbf{F} ; \mathbb{P}_{\eta}(\mathcal{A}) ; \tau_{\eta}^{*}(\mathcal{A}) ; \mathfrak{I} ; \mathfrak{Y}\right]= \\
& =(\operatorname{sas})\left[\mathbf{F} ; \mathbb{P}_{\eta}(\mathcal{A}) ; \tau_{\eta}^{0}(\mathcal{A}) ; \mathfrak{I} ; \widehat{\mathfrak{Y}}\right]=(\operatorname{sas})\left[\mathbf{F} ; \mathbb{P}_{\eta}(\mathcal{A}) ; \tau_{\eta}^{*}(\mathcal{A}) ; \mathfrak{I} ; \widehat{\mathfrak{Y}}\right]
\end{aligned}
$$


Доказательство. В силу предложения 5.1 и (5.35) имеем цепочку вложений

$$
\text { (as) }\left[\mathbf{F} ; \mathbb{P}_{\eta}(\mathcal{A}) ; \tau_{\eta}^{*}(\mathcal{A}) ; \mathfrak{I} ; \mathfrak{Y}\right] \subset \widetilde{\mathbb{P}}_{\eta}^{0}(\mathcal{A}) \subset(\operatorname{sas})\left[\mathbf{F} ; \mathbb{P}_{\eta}(\mathcal{A}) ; \tau_{\eta}^{0}(\mathcal{A}) ; \mathfrak{I} ; \widehat{\mathfrak{Y}}\right]
$$

С учетом (3.3), (5.7), (5.9) и (5.37) получаем, в частности,

$$
\begin{aligned}
\widetilde{\mathbb{P}}_{\eta}^{0}(\mathcal{A}) & \subset(\operatorname{sas})\left[\mathbf{F} ; \mathbb{P}_{\eta}(\mathcal{A}) ; \tau_{\eta}^{0}(\mathcal{A}) ; \mathfrak{I} ; \widehat{\mathfrak{Y}}\right] \subset(\operatorname{sas})\left[\mathbf{F} ; \mathbb{P}_{\eta}(\mathcal{A}) ; \tau_{\eta}^{*}(\mathcal{A}) ; \mathfrak{I} ; \widehat{\mathfrak{Y}}\right] \subset \\
& \subset(\operatorname{sas})\left[\mathbf{F} ; \mathbb{P}_{\eta}(\mathcal{A}) ; \tau_{\eta}^{*}(\mathcal{A}) ; \mathfrak{I} ; \mathfrak{Y}\right] \subset(\operatorname{as})\left[\mathbf{F} ; \mathbb{P}_{\eta}(\mathcal{A}) ; \tau_{\eta}^{*}(\mathcal{A}) ; \mathfrak{I} ; \mathfrak{Y}\right]
\end{aligned}
$$

откуда с учетом (5.37) получаем цепочку равенств

$$
\begin{aligned}
\widetilde{\mathbb{P}}_{\eta}^{0}(\mathcal{A}) & =(\operatorname{sas})\left[\mathbf{F} ; \mathbb{P}_{\eta}(\mathcal{A}) ; \tau_{\eta}^{0}(\mathcal{A}) ; \mathfrak{I} ; \widehat{\mathfrak{Y}}\right]=(\operatorname{sas})\left[\mathbf{F} ; \mathbb{P}_{\eta}(\mathcal{A}) ; \tau_{\eta}^{*}(\mathcal{A}) ; \mathfrak{I} ; \widehat{\mathfrak{Y}}\right]= \\
& =(\operatorname{sas})\left[\mathbf{F} ; \mathbb{P}_{\eta}(\mathcal{A}) ; \tau_{\eta}^{*}(\mathcal{A}) ; \mathfrak{I} ; \mathfrak{Y}\right]=(\operatorname{as})\left[\mathbf{F} ; \mathbb{P}_{\eta}(\mathcal{A}) ; \tau_{\eta}^{*}(\mathcal{A}) ; \mathfrak{I} ; \mathfrak{Y}\right]
\end{aligned}
$$

В дополнение к (5.38) отметим, что согласно (5.7) по определению $\mathfrak{Y}, \widehat{\mathfrak{Y}}$ имеем вложения

$$
\text { (as) }\left[\mathbf{F} ; \mathbb{P}_{\eta}(\mathcal{A}) ; \tau_{\eta}^{*}(\mathcal{A}) ; \mathfrak{I} ; \widehat{\mathfrak{Y}}\right] \subset(\operatorname{as})\left[\mathbf{F} ; \mathbb{P}_{\eta}(\mathcal{A}) ; \tau_{\eta}^{*}(\mathcal{A}) ; \mathfrak{I} ; \mathfrak{Y}\right]
$$

Из (5.38), (5.39) следует включение $(\operatorname{as})\left[\mathbf{F} ; \mathbb{P}_{\eta}(\mathcal{A}) ; \tau_{\eta}^{*}(\mathcal{A}) ; \mathfrak{I} ; \widehat{\mathfrak{Y}}\right] \subset \widetilde{\mathbb{P}}_{\eta}^{0}(\mathcal{A})$. С другой стороны, согласно (3.3) и (5.38) имеем

$$
\widetilde{\mathbb{P}}_{\eta}^{0}(\mathcal{A})=(\operatorname{sas})\left[\mathbf{F} ; \mathbb{P}_{\eta}(\mathcal{A}) ; \tau_{\eta}^{*}(\mathcal{A}) ; \mathfrak{I} ; \widehat{\mathfrak{Y}}\right] \subset(\operatorname{as})\left[\mathbf{F} ; \mathbb{P}_{\eta}(\mathcal{A}) ; \tau_{\eta}^{*}(\mathcal{A}) ; \mathfrak{I} ; \widehat{\mathfrak{Y}}\right],
$$

а потому получаем следующее равенство:

$$
\widetilde{\mathbb{P}}_{\eta}^{0}(\mathcal{A})=(\operatorname{as})\left[\mathbf{F} ; \mathbb{P}_{\eta}(\mathcal{A}) ; \tau_{\eta}^{*}(\mathcal{A}) ; \mathfrak{I} ; \widehat{\mathfrak{Y}}\right]
$$

С учетом (3.3) получаем также

$$
(\operatorname{sas})\left[\mathbf{F} ; \mathbb{P}_{\eta}(\mathcal{A}) ; \tau_{\eta}^{0}(\mathcal{A}) ; \mathfrak{I} ; \widehat{\mathfrak{Y}}\right] \subset(\operatorname{as})\left[\mathbf{F} ; \mathbb{P}_{\eta}(\mathcal{A}) ; \tau_{\eta}^{0}(\mathcal{A}) ; \mathfrak{I} ; \widehat{\mathfrak{Y}}\right]
$$

Из $(5.38)$ и $(5.41)$ имеем $\widetilde{\mathbb{P}}_{\eta}^{0}(\mathcal{A}) \subset(\operatorname{as})\left[\mathbf{F} ; \mathbb{P}_{\eta}(\mathcal{A}) ; \tau_{\eta}^{0}(\mathcal{A}) ; \mathfrak{I} ; \widehat{\mathfrak{Y}}\right]$, где (см. $(5.9)$ и $\left.(5.40)\right)$

$$
(\operatorname{as})\left[\mathbf{F} ; \mathbb{P}_{\eta}(\mathcal{A}) ; \tau_{\eta}^{0}(\mathcal{A}) ; \mathfrak{I} ; \widehat{\mathfrak{Y}}\right] \subset(\operatorname{as})\left[\mathbf{F} ; \mathbb{P}_{\eta}(\mathcal{A}) ; \tau_{\eta}^{*}(\mathcal{A}) ; \mathfrak{I} ; \widehat{\mathfrak{Y}}\right]=\widetilde{\mathbb{P}}_{\eta}^{0}(\mathcal{A}),
$$

а тогда справедливо равенство

$$
\widetilde{\mathbb{P}}_{\eta}^{0}(\mathcal{A})=(\operatorname{as})\left[\mathbf{F} ; \mathbb{P}_{\eta}(\mathcal{A}) ; \tau_{\eta}^{0}(\mathcal{A}) ; \mathfrak{\Im} ; \widehat{\mathfrak{Y}}\right]
$$

Далее, с учетом (5.7) и определения семейств $\mathfrak{Y}, \widehat{\mathfrak{Y}}$ получаем

$$
\text { (as) }\left[\mathbf{F} ; \mathbb{P}_{\eta}(\mathcal{A}) ; \tau_{\eta}^{0}(\mathcal{A}) ; \mathfrak{I} ; \widehat{\mathfrak{Y}}\right] \subset(\operatorname{as})\left[\mathbf{F} ; \mathbb{P}_{\eta}(\mathcal{A}) ; \tau_{\eta}^{0}(\mathcal{A}) ; \mathfrak{I} ; \mathfrak{Y}\right],
$$

а тогда (см. $(5.42)) \widetilde{\mathbb{P}}_{\eta}^{0}(\mathcal{A}) \subset(\operatorname{as})\left[\mathbf{F} ; \mathbb{P}_{\eta}(\mathcal{A}) ; \tau_{\eta}^{0}(\mathcal{A}) ; \mathfrak{I} ; \mathfrak{Y}\right]$, где (см. $(5.9)$ и (5.38))

$$
(\operatorname{as})\left[\mathbf{F} ; \mathbb{P}_{\eta}(\mathcal{A}) ; \tau_{\eta}^{0}(\mathcal{A}) ; \mathfrak{I} ; \mathfrak{Y}\right] \subset(\operatorname{as})\left[\mathbf{F} ; \mathbb{P}_{\eta}(\mathcal{A}) ; \tau_{\eta}^{*}(\mathcal{A}) ; \mathfrak{I} ; \mathfrak{Y}\right]=\widetilde{\mathbb{P}}_{\eta}^{0}(\mathcal{A})
$$

Как следствие получаем очевидное равенство

$$
\widetilde{\mathbb{P}}_{\eta}^{0}(\mathcal{A})=(\operatorname{as})\left[\mathbf{F} ; \mathbb{P}_{\eta}(\mathcal{A}) ; \tau_{\eta}^{0}(\mathcal{A}) ; \mathfrak{\Im} ; \mathfrak{Y}\right] .
$$

Поскольку (см. (5.7)) (sas) $\left[\mathbf{F} ; \mathbb{P}_{\eta}(\mathcal{A}) ; \tau_{\eta}^{0}(\mathcal{A}) ; \mathfrak{\Im} ; \widehat{\mathfrak{Y}}\right] \subset(\operatorname{sas})\left[\mathbf{F} ; \mathbb{P}_{\eta}(\mathcal{A}) ; \tau_{\eta}^{0}(\mathcal{A}) ; \mathfrak{I} ; \mathfrak{Y}\right]$, из $(5.38)$ имеем

$$
\widetilde{\mathbb{P}}_{\eta}^{0}(\mathcal{A}) \subset(\operatorname{sas})\left[\mathbf{F} ; \mathbb{P}_{\eta}(\mathcal{A}) ; \tau_{\eta}^{0}(\mathcal{A}) ; \mathfrak{I} ; \mathfrak{Y}\right] .
$$


Но $($ см. $(5.9))($ sas $)\left[\mathbf{F} ; \mathbb{P}_{\eta}(\mathcal{A}) ; \tau_{\eta}^{0}(\mathcal{A}) ; \mathfrak{I} ; \mathfrak{Y}\right] \subset(\operatorname{sas})\left[\mathbf{F} ; \mathbb{P}_{\eta}(\mathcal{A}) ; \tau_{\eta}^{*}(\mathcal{A}) ; \mathfrak{I} ; \mathfrak{Y}\right]$, а потому (см. (5.38)) $(\mathrm{sas})\left[\mathbf{F} ; \mathbb{P}_{\eta}(\mathcal{A}) ; \tau_{\eta}^{0}(\mathcal{A}) ; \mathfrak{I} ; \mathfrak{Y}\right] \subset \widetilde{\mathbb{P}}_{\eta}^{0}(\mathcal{A})$ и с учетом $(5.44)$ реализуется равенство

$$
\widetilde{\mathbb{P}}_{\eta}^{0}(\mathcal{A})=(\operatorname{sas})\left[\mathbf{F} ; \mathbb{P}_{\eta}(\mathcal{A}) ; \tau_{\eta}^{0}(\mathcal{A}) ; \mathfrak{I} ; \mathfrak{Y}\right]
$$

Из (5.38), (5.40), (5.42), (5.43) и (5.45) вытекает (5.36).

В теореме 5.1 утверждается, что $\widetilde{\mathbb{P}}_{\eta}^{0}(\mathcal{A})$ есть вспомогательное МП, обладающее существенной универсальностью как в части используемых топологий, так и в части применяемых ОАХ.

\section{6. МНОЖЕСТВО ПРИТЯЖЕНИЯ В КОНЕЧНОМЕРНОМ ПРОСТРАНСТВЕ}

В данном разделе рассматривается конструкция, ориентированная на построение асимптотического аналога ОД в заданный момент времени; при этом имеется в виду ОД линейной системы с разрывностью в коэффициентах при управляющих воздействиях, в результате чего появляется возможность возникновения эффектов типа произведения разрывной функции на обобщенную. Существенная часть построения ОД и МП (в качестве асимптотического аналога ОД) касается работы с интегрантом скалярного (в нашем случае) управления. По этой причине в дальнейшем исследуется только упомянутый интегрант, его возможные значения в виде соответствующих векторов, а также элементы притяжения в классе таких векторов. Переход к рассмотрению самой ОД и ее асимптотических аналогов в виде МП осуществляется очевидным образом с использованием сдвига, определяемого начальным состоянием системы. Кроме того, данная форма изложения делает возможным другие интерпретации используемых процедур.

Фиксируем $n \in \mathbb{N}$ и кортеж $\left(\pi_{i}\right)_{i \in \overline{1, n}}: \overline{1, n} \rightarrow B(I, \mathcal{A}) ;$ итак, $\pi_{j} \in B(I, \mathcal{A})$ при $j \in \overline{1, n}$. Мы имеем, таким образом, набор ярусных функций, порождающих вектор-функцию со значениями в $\mathbb{R}^{n}$, т.е. $n$-вектор-функцию. Введем в рассмотрение отображение П вида

$$
f \mapsto\left(\int_{I} \pi_{i} f d \eta\right)_{i \in \overline{1, n}}: \quad \mathbf{F} \rightarrow \mathbb{R}^{n}
$$

(П есть обычный вектор-функционал на $\mathbf{F}) ;$ итак, $\Pi: \mathbf{F} \rightarrow \mathbb{R}^{n}$. Тогда при $\mathcal{H} \in \beta[\mathbf{F}]$ определено МП

$$
\text { (as) }\left[\mathbf{F} ; \mathbb{R}^{n} ; \tau_{\mathbb{R}}^{(n)} ; \Pi ; \mathcal{H}\right]=\bigcap_{H \in \mathcal{H}} \operatorname{cl}\left(\Pi^{1}(H), \tau_{\mathbb{R}}^{(n)}\right) \in \mathcal{P}\left(\mathbb{R}^{n}\right),
$$

где $\tau_{\mathbb{R}}^{(n)}$ - обычная топология покоординатной сходимости в $\mathbb{R}^{n}$. В частности, в качестве $\mathcal{H}$ можно использовать $\mathfrak{Y}$ и $\widehat{\mathfrak{Y}}$. В этих случаях МП (6.1) может также быть полезным для построения расширений задачи о нахождении ОД, “заменяя" множество возможных значений интегранта управления. Пусть П определено как отображение

$$
\mu \mapsto\left(\int_{I} \pi_{i} d \mu\right)_{i \in \overline{1, n}}: \quad \mathbb{P}_{\eta}(\mathcal{A}) \rightarrow \mathbb{R}^{n}
$$

( $\widetilde{\Pi}$ есть вектор-функционал на $\mathbb{P}_{\eta}(\mathcal{A})$ ). По свойствам $\mathfrak{I}$ имеем очевидное равенство

$$
\Pi=\widetilde{\Pi} \circ \mathfrak{I}
$$

где (по свойствам *-слабой топологии)

$$
\widetilde{\Pi} \in C\left(\mathbb{P}_{\eta}(\mathcal{A}), \tau_{\eta}^{*}(\mathcal{A}), \mathbb{R}^{n}, \tau_{\mathbb{R}}^{(n)}\right) .
$$


Поскольку $\left(\mathbb{P}_{\eta}(\mathcal{A}), \tau_{\eta}^{*}(\mathcal{A})\right)$ - непустой компакт, с учетом $(3.4),(6.2),(6.3)$ имеем следующее представление интересующих нас МП в $\mathbb{R}^{n}$ (см. также теорему 5.1):

$$
(\mathrm{as})\left[\mathbf{F} ; \mathbb{R}^{n} ; \tau_{\mathbb{R}}^{(n)} ; \Pi ; \widehat{\mathfrak{Y}}\right]=(\operatorname{as})\left[\mathbf{F} ; \mathbb{R}^{n} ; \tau_{\mathbb{R}}^{(n)} ; \Pi ; \mathfrak{Y}\right]=\widetilde{\Pi}^{1}\left(\widetilde{\mathbb{P}}_{\eta}^{0}(\mathcal{A})\right)
$$

Теорема 6.1. Имеет место цепочка равенств

$$
\begin{aligned}
\widetilde{\Pi}^{1}\left(\widetilde{\mathbb{P}}_{\eta}^{0}(\mathcal{A})\right) & =(\operatorname{as})\left[\mathbf{F} ; \mathbb{R}^{n} ; \tau_{\mathbb{R}}^{(n)} ; \Pi ; \widehat{\mathfrak{Y}}\right]=(\operatorname{as})\left[\mathbf{F} ; \mathbb{R}^{n} ; \tau_{\mathbb{R}}^{(n)} ; \Pi ; \mathfrak{Y}\right]= \\
& =(\text { sas })\left[\mathbf{F} ; \mathbb{R}^{n} ; \tau_{\mathbb{R}}^{(n)} ; \Pi ; \widehat{\mathfrak{Y}}\right]=(\text { sas })\left[\mathbf{F} ; \mathbb{R}^{n} ; \tau_{\mathbb{R}}^{(n)} ; \Pi ; \mathfrak{Y}\right]
\end{aligned}
$$

Для доказательства достаточно учесть (6.4) и дополнительно отметить, что (см. [6, Proposition 3.3.1])

$$
\begin{aligned}
& (\text { as })\left[\mathbf{F} ; \mathbb{R}^{n} ; \tau_{\mathbb{R}}^{(n)} ; \Pi ; \widehat{\mathfrak{Y}}\right]=(\operatorname{sas})\left[\mathbf{F} ; \mathbb{R}^{n} ; \tau_{\mathbb{R}}^{(n)} ; \Pi ; \widehat{\mathfrak{Y}}\right] \\
& (\text { as })\left[\mathbf{F} ; \mathbb{R}^{n} ; \tau_{\mathbb{R}}^{(n)} ; \Pi ; \mathfrak{Y}\right]=(\operatorname{sas})\left[\mathbf{F} ; \mathbb{R}^{n} ; \tau_{\mathbb{R}}^{(n)} ; \Pi ; \mathfrak{Y}\right]
\end{aligned}
$$

Из теоремы 6.1 следует, что достаточные условия асимптотической нечувствительности при ослаблении части ограничений, подобные исследуемым в $[5-7,9,23]$ (и в целом ряде других работ), сохраняют свою силу и в режиме "узких импульсов". Данное свойство (см. теорему 6.1) может быть полезным в вопросах практической реализации импульсных управлений. С учетом теоремы 6.1 основное внимание в дальнейшем уделяется вопросам представления множества $\widetilde{\Pi}^{1}\left(\widetilde{\mathbb{P}}_{\eta}^{0}(\mathcal{A})\right)$, являющегося своеобразным "универсальным" МП. Будем использовать (5.22), (5.28). Тогда, в частности,

$$
\begin{array}{ll}
\int_{I} \pi_{j} d \varkappa\left(\mathcal{U}_{t}^{(-)}\right)=\lim _{\theta \uparrow t} \pi_{j}(\theta) & \forall t \in] a, b] \quad \forall j \in \overline{1, n}, \\
\int_{I} \pi_{j} d \varkappa\left(\mathcal{U}_{t}^{(+)}\right)=\lim _{\theta \downarrow t} \pi_{j}(\theta) & \forall t \in[a, b[\quad \forall j \in \overline{1, n} .
\end{array}
$$

Имея в виду эти соотношения, введем в рассмотрение вектор-функции, отвечающие операциям односторонних пределов: определим $\vec{\pi}:] a, b] \rightarrow \mathbb{R}^{n}$ правилом

$$
\left.\left.\vec{\pi}(t) \triangleq\left(\lim _{\theta \uparrow t} \pi_{i}(\theta)\right)_{i \in \overline{1, n}} \quad \forall t \in\right] a, b\right]
$$

а $\overleftarrow{\pi}:\left[a, b\left[\rightarrow \mathbb{R}^{n}\right.\right.$ правилом

$$
\overleftarrow{\pi}(t) \triangleq\left(\lim _{\theta \downarrow t} \pi_{i}(\theta)\right)_{i \in \overline{1, n}} \quad \forall t \in[a, b[
$$

Тогда получаем следующие очевидные представления:

$$
\left.\left.\widetilde{\Pi}\left(\varkappa\left(\mathcal{U}_{t}^{(-)}\right)\right)=\vec{\pi}(t) \quad \forall t \in\right] a, b\right], \quad \widetilde{\Pi}\left(\varkappa\left(\mathcal{U}_{t}^{(+)}\right)\right)=\overleftarrow{\pi}(t) \quad \forall t \in[a, b[.
$$

Напомним (5.24), (5.30), а также следующее легко проверяемое свойство:

$$
\begin{gathered}
\forall t \in] a, b[\quad \forall \alpha \in[0,1] \\
\left(\alpha \varkappa\left(\mathcal{U}_{t}^{(-)}\right)+(1-\alpha) \varkappa\left(\mathcal{U}_{t}^{(+)}\right) \in \widetilde{\mathbb{P}}_{\eta}^{0}(\mathcal{A})\right) \Leftrightarrow\left(\alpha \widehat{\rho} \uparrow(t)+(1-\alpha) \widehat{\rho}_{\downarrow}(t) \in \mathbf{Y}\right) .
\end{gathered}
$$


В этой связи введем в рассмотрение множество

$$
\Gamma \triangleq\{z \in] a, b\left[\times[0,1] \mid \operatorname{pr}_{2}(z) \widehat{\rho}_{\uparrow}\left(\operatorname{pr}_{1}(z)\right)+\left(1-\operatorname{pr}_{2}(z)\right) \widehat{\rho}_{\downarrow}\left(\operatorname{pr}_{1}(z)\right) \in \mathbf{Y}\right\}
$$

(см. обозначения разд. 2). Итак, элементами $\Gamma \in \mathcal{P}(] a, b[\times[0,1])$ являются УП $(t, \alpha), t \in] a, b[$, $\alpha \in[0,1]$, для которых реализуется утверждение в правой части (6.6), и только они. Из соотношений (6.5)-(6.7) легко следует, что

$$
\Omega \triangleq\left\{\operatorname{pr}_{2}(z) \vec{\pi}\left(\operatorname{pr}_{1}(z)\right)+\left(1-\operatorname{pr}_{2}(z)\right) \overleftarrow{\pi}\left(\operatorname{pr}_{1}(z)\right): z \in \Gamma\right\} \in \mathcal{P}\left(\widetilde{\Pi}^{1}\left(\widetilde{\mathbb{P}}_{\eta}^{0}(\mathcal{A})\right)\right)
$$

Итак (см. (6.7), (6.8)), если $t \in] a, b[$ и $\alpha \in[0,1]$, то

$$
\left(\alpha \widehat{\rho} \uparrow(t)+(1-\alpha) \widehat{\rho}_{\downarrow}(t) \in \mathbf{Y}\right) \Rightarrow\left(\alpha \vec{\pi}(t)+(1-\alpha) \overleftarrow{\pi}(t) \in \widetilde{\Pi}^{1}\left(\widetilde{\mathbb{P}}_{\eta}^{0}(\mathcal{A})\right)\right)
$$

Далее, из (5.24), (6.5) и теоремы 5.1 вытекает, что

$$
\left(\widehat{\rho}_{\uparrow}(b) \in \mathbf{Y}\right) \Rightarrow\left(\vec{\pi}(b) \in \widetilde{\Pi}^{1}\left(\widetilde{\mathbb{P}}_{\eta}^{0}(\mathcal{A})\right)\right) .
$$

В свою очередь, из (5.30), (6.5) и теоремы 5.1 следует, что

$$
\left(\widehat{\rho}_{\downarrow}(a) \in \mathbf{Y}\right) \Rightarrow\left(\overleftarrow{\pi}(a) \in \widetilde{\Pi}^{1}\left(\widetilde{\mathbb{P}}_{\eta}^{0}(\mathcal{A})\right)\right)
$$

Теперь (см. (5.2), (5.8), (6.9)-(6.11)) получаем исчерпывающее описание МП в $\mathbb{R}^{n}$.

Теорема 6.2. Основное МП $\widetilde{\Pi}^{1}\left(\widetilde{\mathbb{P}}_{\eta}^{0}(\mathcal{A})\right)$ определяется следующими условиями:

1) если $\widehat{\rho}_{\uparrow}(b) \notin \mathbf{Y} u \widehat{\rho}_{\downarrow}(a) \notin \mathbf{Y}, m o \widetilde{\Pi}^{1}\left(\widetilde{\mathbb{P}}_{\eta}^{0}(\mathcal{A})\right)=\Omega$;

2) если $\widehat{\rho}_{\uparrow}(b) \in \mathbf{Y}$ u $\widehat{\rho}_{\downarrow}(a) \notin \mathbf{Y}$, mo $\widetilde{\Pi}^{1}\left(\widetilde{\mathbb{P}}_{\eta}^{0}(\mathcal{A})\right)=\Omega \cup\{\vec{\pi}(b)\}$;

3) если $\widehat{\rho}_{\uparrow}(b) \notin \mathbf{Y}$ u $\widehat{\rho}_{\downarrow}(a) \in \mathbf{Y}$, mo $\widetilde{\Pi}^{1}\left(\widetilde{\mathbb{P}}_{\eta}^{0}(\mathcal{A})\right)=\Omega \cup\{\overleftarrow{\pi}(a)\}$

4) если $\widehat{\rho}_{\uparrow}(b) \in \mathbf{Y} u \widehat{\rho}_{\downarrow}(a) \in \mathbf{Y}$, mo $\widetilde{\Pi}^{1}\left(\widetilde{\mathbb{P}}_{\eta}^{0}(\mathcal{A})\right)=\Omega \cup\{\overleftarrow{\pi}(a) ; \vec{\pi}(b)\}$.

Подчеркнем, что согласно теореме $6.2 \mathrm{MП}$, рассматриваемое в теореме 6.1 и обладающее определенной универсальностью относительно конкретного выбора ОАХ, полностью определяется (см. (6.7), (6.8)) односторонними пределами функций $\rho_{1}, \ldots, \rho_{N}, \pi_{1}, \ldots, \pi_{n}$, заданных по условиям задачи.

\section{7. ДОБАВЛЕНИЕ}

В настоящем кратком разделе приведены полезные следствия теоремы 6.1. Речь идет о реализации МП и самого свойства асимптотической нечувствительности в терминах окрестностей. Мы оснащаем для определенности $\mathbb{R}^{n}$ нормой $\|\cdot\|_{n}$ вида

$$
\left(x_{i}\right)_{i \in \overline{1, n}} \mapsto \max _{1 \leq i \leq n}\left|x_{i}\right|: \quad \mathbb{R}^{n} \rightarrow[0, \infty[
$$

Если $H \in \mathcal{P}\left(\mathbb{R}^{n}\right)$ и $\left.\varepsilon \in\right] 0, \infty\left[\right.$, то $\mathbb{O}(H, \varepsilon) \triangleq\left\{z \in \mathbb{R}^{n} \mid \exists h \in H:\|h-z\|_{n}<\varepsilon\right\} \in \tau_{\mathbb{R}}^{(n)}$ (открытая $\varepsilon$-окрестность $H$ ). Из теоремы 6.1 и [23, Proposition 3.6.1] (последнее - простое следствие [26, предложение 3.5.1]) вытекает, что $\forall \xi \in] 0, \infty[\exists \varepsilon \in] 0, \infty[\forall \delta \in] 0, \varepsilon[$

$$
\begin{aligned}
& \widetilde{\Pi}^{1}\left(\widetilde{\mathbb{P}}_{\eta}^{0}(\mathcal{A})\right) \subset \operatorname{cl}\left(\Pi^{1}\left(\mathbb{Y}_{\delta}\right), \tau_{\mathbb{R}}^{(n)}\right) \subset \mathbb{O}\left(\widetilde{\Pi}^{1}\left(\widetilde{\mathbb{P}}_{\eta}^{0}(\mathcal{A})\right), \xi\right), \\
& \widetilde{\Pi}^{1}\left(\widetilde{\mathbb{P}}_{\eta}^{0}(\mathcal{A})\right) \subset \operatorname{cl}\left(\Pi^{1}\left(\widehat{\mathbb{Y}}_{\delta}\right), \tau_{\mathbb{R}}^{(n)}\right) \subset \mathbb{O}\left(\widetilde{\Pi}^{1}\left(\widetilde{\mathbb{P}}_{\eta}^{0}(\mathcal{A})\right), \xi\right) .
\end{aligned}
$$


Здесь мы учли тот очевидный факт, что $\Pi^{1}(\mathbf{F}) \subset \widetilde{\Pi}^{1}\left(\mathbb{P}_{\eta}(\mathcal{A})\right)$, где $\widetilde{\Pi}^{1}\left(\mathbb{P}_{\eta}(\mathcal{A})\right) \in\left(\tau_{\mathbb{R}}^{(n)}\right.$-comp $)\left[\mathbb{R}^{n}\right]$ (см. (6.3)), а тогда $\operatorname{cl}\left(\Pi^{1}(H), \tau_{\mathbb{R}}^{(n)}\right) \in\left(\tau_{\mathbb{R}}^{(n)}\right.$-comp) $\left[\mathbb{R}^{n}\right] \cup\{\varnothing\}$ при $H \in \mathfrak{Y} \cup \widehat{\mathfrak{Y}}$. Свойство $(7.1)$ означает, что универсальное МП в теореме 6.1 (определяемое явным образом в теореме 6.2) для обоих типов ослабления "моментных" ограничений реализует асимптотику “обычных" ОД, получаемых в режиме "узких импульсов", с точностью до любой наперед выбранной окрестности. Рассмотрим теперь естественный аналог положения [5, Theorem 6.3.3] в виде простого следствия (7.1).

Предложение 7.1. Если $\xi \in] 0, \infty[$, то

$$
\exists \varepsilon \in] 0, \infty[\quad \forall \delta \in] 0, \varepsilon\left[\quad \Pi^{1}\left(\widehat{\mathbb{Y}}_{\delta}\right) \subset \Pi^{1}\left(\mathbb{Y}_{\delta}\right) \subset \mathbb{O}\left(\Pi^{1}\left(\widehat{\mathbb{Y}}_{\delta}\right), \xi\right) .\right.
$$

Доказательство. Учитываем (5.7). Используя (7.1), подберем $\left.\varepsilon_{0} \in\right] 0, \infty[$ так, что при этом

$$
\left.\widetilde{\Pi}^{1}\left(\widetilde{\mathbb{P}}_{\eta}^{0}(\mathcal{A})\right) \subset \operatorname{cl}\left(\Pi^{1}\left(\mathbb{Y}_{\zeta}\right), \tau_{\mathbb{R}}^{(n)}\right) \subset \mathbb{O}\left(\widetilde{\Pi}^{1}\left(\widetilde{\mathbb{P}}_{\eta}^{0}(\mathcal{A})\right), \frac{\xi}{2}\right) \quad \forall \zeta \in\right] 0, \varepsilon_{0}[.
$$

Пусть $\delta \in] 0, \varepsilon_{0}\left[\right.$. Выберем произвольно $z_{0} \in \Pi^{1}\left(\mathbb{Y}_{\delta}\right)$ и с учетом $(7.2)$ подберем $y_{0} \in \widetilde{\Pi}^{1}\left(\widetilde{\mathbb{P}}_{\eta}^{0}(\mathcal{A})\right)$ так, что при этом

$$
\left\|y_{0}-z_{0}\right\|_{n}<\frac{\xi}{2}
$$

Тогда, так как (см. (6.1), теорема 6.1) $\widetilde{\Pi}^{1}\left(\widetilde{\mathbb{P}}_{\eta}^{0}(\mathcal{A})\right) \subset \operatorname{cl}\left(\Pi^{1}\left(\widehat{\mathbb{Y}}_{\delta}\right), \tau_{\mathbb{R}}^{(n)}\right)$, имеем $y_{0} \in \operatorname{cl}\left(\Pi^{1}\left(\widehat{\mathbb{Y}}_{\delta}\right), \tau_{\mathbb{R}}^{(n)}\right)$, а тогда для некоторого $y^{0} \in \Pi^{1}\left(\widehat{\mathbb{Y}}_{\delta}\right)$

$$
\left\|y_{0}-y^{0}\right\|<\frac{\xi}{2}
$$

С учетом (7.3) получаем $\left\|y^{0}-z_{0}\right\|_{n}<\xi$. Это означает, что $z_{0} \in \mathbb{O}\left(\Pi^{1}\left(\widehat{\mathbb{Y}}_{\delta}\right), \xi\right)$. Коль скоро выбор $z_{0}$ был произвольным, получаем $\Pi^{1}\left(\mathbb{Y}_{\delta}\right) \subset \mathbb{O}\left(\Pi^{1}\left(\widehat{\mathbb{Y}}_{\delta}\right), \xi\right)$. Поскольку $\Pi^{1}\left(\widehat{\mathbb{Y}}_{\delta}\right) \subset \Pi^{1}\left(\mathbb{Y}_{\delta}\right)$, требуемое свойство установлено.

Предложение 7.1 реализует свойство асимптотической нечувствительности (при ослаблении части ограничений) в "окрестностной" форме. Само это свойство связано со ступенчатостью (точнее, $\mathcal{A}$-ступенчатостью) некоторых в/з функций (5.3), являющихся компонентами $N$-вектор-функции, определяющей матрицант в (5.4). Иными словами, речь идет о случае $M \neq \varnothing($ см. $(5.5))$.

Отметим простейший пример, касающийся управления материальной точкой

$$
\dot{x}_{1}(t)=x_{2}(t), \quad \dot{x}_{2}(t)=f(t) \widetilde{b}(t)
$$

на промежутке $[0,1]$ при нулевых начальных условиях $x_{1}(0)=x_{2}(0)=0$; полагаем, что в (7.4) $\widetilde{b}=\widetilde{b}(\cdot 2-$ неубывающая к.п. в/з функция. Полагая также $a=0$ и $b=1$, получаем $I=[0,1]$; тогда $\vec{b} \in B_{0}(I, \mathcal{A})$. Фиксируем $t_{1}, t_{2} \in I$ и рассматриваем условие

$$
\left(x_{1}\left(t_{1}\right), x_{2}\left(t_{2}\right)\right) \in \mathbf{Y}
$$

где $x(\cdot)=\left(x_{1}(\cdot), x_{2}(\cdot)\right)$ - траектория, порождаемая управлением из $\mathbf{F}$ (см. (4.5) при $a=0$, $b=1)$, а $\mathbf{Y}-$ непустое замкнутое множество на плоскости. С учетом формулы Коши (7.5) легко сводится к условию (5.4), в котором (для $N=2$ ) при $t \in I$

$$
\rho_{1}(t)=\left(t_{1}-t\right) \widetilde{b}(t) \chi_{\left[0, t_{1}[\right.}(t), \quad \rho_{2}(t)=\widetilde{b}(t) \chi_{\left[0, t_{2}[\right.}(t) .
$$


Ясно, что $\rho_{2} \in B_{0}(I, \mathcal{A})$ и в качестве $M$ можно выбрать синглетон $\{2\}$. В настоящей работе данный (неоднократно рассматривавшийся ранее) пример модифицирован для исследования режима "узких импульсов", что является новым обстоятельством. Заметим, что точки разрыва функции $\widetilde{b}$ соответствуют по смыслу моментам сброса массы.

Отметим, что постановка задачи в настоящей статье усложнена в сравнении с работой [22] за счет использования асимптотического аналога условия (5.4), что, в частности, отражено в последнем примере.

\section{СПИСОК ЛИТЕРАТУРЫ}

1. Bhaskara Rao K.P.S., Bhaskara Rao M. Theory of charges: A study of finitely additive measures. London: Acad. Press, 1983.

2. Бурбаки Н. Общая топология: Основные структуры. М.: Наука, 1968.

3. Булинский А.В., Ширяев А.Н. Теория случайных процессов. М.: Физматлит, 2005.

4. Ченцов А.Г. К вопросу о корректном расширении одной задачи о выборе плотности вероятности при ограничениях на систему математических ожиданий // УМН. 1995. Т. 50, № 5. С. 223-242.

5. Chentsov A.G. Finitely additive measures and relaxations of extremal problems. New York: Plenum Publ., 1996.

6. Chentsov A.G. Asymptotic attainability. Dordrecht: Kluwer, 1997.

7. Ченцов А.Г. К вопросу о корректном расширении некоторых неустойчивых задач управления с интегральными ограничениями // Изв. РАН. Сер. мат. 1999. Т. 63, № 3. С. 185-223.

8. Ченцов А.Г. К вопросу о корректном расширении некоторых неустойчивых задач обработки статистической информации // Кибернетика и сист. анализ. 2001. № 2. С. 110-131.

9. Chentsov A.G. Finitely additive measures and extensions of abstract control problems // J. Math. Sci. 2006. V. 133, N 2. P. 1045-1206.

10. Ченцов А.Г. Элементы конечно-аддитивной теории меры. Екатеринбург: УГТУ-УПИ, 2008, 2010. Т. 1, 2.

11. Ченцов А.Г. Фильтры и ультрафильтры в конструкциях множеств притяжения // Вестн. Удмурт. ун-та. Математика. Механика. Компьют. науки. 2011. № 1. С. 113-142.

12. Ченцов А.Г. Ультрафильтры измеримых пространств как обобщенные решения в абстрактных задачах о достижимости // Тр. Ин-та математики и механики УрО РАН. 2011. Т. 17, № 1. С. 268-293.

13. Ченцов А.Г. Об одном примере представления пространства ультрафильтров алгебры множеств // Тр. Ин-та математики и механики УрО РАН. 2011. Т. 17, № 4. С. 293-311.

14. Ченцов А.Г. Ярусные отображения и преобразования на основе ультрафильтров // Тр. Ин-та математики и механики УрО РАН. 2012. Т. 18, № 4. С. 298-314.

15. Ченцов А.Г. К вопросу о представлении элементов притяжения в абстрактных задачах о достижимости с ограничениями асимптотического характера // Изв. вузов. Математика. 2012. № 10. С. 45-59.

16. Ченцов А.Г. К вопросу о структуре множеств притяжения в топологическом пространстве // Изв. Ин-та математики и информатики УдГУ. 2012. №1. С. 147-150.

17. Ченцов А.Г. Множества притяжения в абстрактных задачах о достижимости: эквивалентные представления и основные свойства // Изв. вузов. Математика. 2013. № 11. С. 33-50.

18. Ченцов А.Г. К вопросу о представлении ультрафильтров и их применении в конструкциях расширений // Тр. Ин-та математики и механики УрО РАН. 2013. Т. 19, № 4. С. 289-307.

19. Ченцов А.Г. K вопросу о представлении компактов Стоуна // Вестн. Удмурт. ун-та. Математика. Механика. Компьют. науки. 2013. № 4. С. 156-174.

20. Ченцов А.Г. О некоторых вопросах структуры ультрафильтров, связанных с расширениями абстрактных задач управления // Автоматика и телемеханика. 2013. № 12. С. 119-139.

21. Ченщов А.Г. Ультрафильтры измеримых пространств и их применение в конструкциях расширений // Тр. Ин-та математики и механики УрО РАН. 2014. Т. 20, № 1. С. 285-304.

22. Ченцов А.Г., Бакланов А.П. К вопросу о построении множества достижимости при ограничениях асимптотического характера // Тр. Ин-та математики и механики УрО РАН. 2014. Т. 20, № 3. С. 309-323.

23. Chentsov A.G., Morina S.I. Extensions and relaxations. Dordrecht: Kluwer, 2002.

24. Данфорд Н., Швари, Дж. Линейные операторы: Общая теория. М.: Изд-во иностр. лит., 1962.

25. Эльясберг П.Е. Введение в теорию полета искусственных спутников Земли. М.: Наука, 1965.

26. Энгелъкинг Р. Общая топология. М.: Мир, 1986.

27. Гамкрелидзе Р.В. Основы оптимального управления. Тбилиси: Изд-во Тбил. ун-та, 1977.

28. Келли Дж.Л. Общая топология. М.: Наука, 1968.

29. Красовский Н.Н. Теория управления движением: Линейные системы. М.: Наука, 1968. 
30. Красовский Н.Н. Дифференциальная игра сближения-уклонения. I, II // Изв. АН СССР. Техн. кибернетика. 1973. № 2. С. 3-18; №3. С. 22-42.

31. Красовский Н.Н., Субботин А.И. Позиционные дифференциальные игры. М.: Наука, 1974.

32. Куратовский К., Мостовский А. Теория множеств. М.: Мир, 1970.

33. Невё Ж. Математические основы теории вероятностей. М.: Мир, 1969.

34. Субботин А.И., Ченцов А.Г. Оптимизация гарантии в задачах управления. М.: Наука, 1981.

35. Понтрягин Л.С., Болтянский В.Г., Гамкрелидзе Р.В., Мищенко Е.Ф. Математическая теория оптимальных процессов. М.: Наука, 1983.

36. Варга Дж. Оптимальное управление дифференциальными и функциональными уравнениями. М.: Наука, 1977. 